Title: BMP signaling is necessary and sufficient for osteoarthritis and a target for disease modifying therapy

\title{
AUTHORS:
}

Akrit Pran Jaswal ${ }^{1}$, Anke J. Roelofs ${ }^{3, \#}$, Amaresh Kumar Singh ${ }^{1,4, \#}$, Bhupendra Kumar ${ }^{1}$, Anna H.K. Riemen $^{3}$, Hui Wang ${ }^{3}$, Sadaf Ashraf ${ }^{3}$, Sanap Vaibhav Nanasaheb ${ }^{1}$, Sayeda Fauzia Iqbal ${ }^{1}$, Nitin Agnihotri ${ }^{1}$, Cosimo De Bari ${ }^{3}$, Amitabha Bandyopadhyay ${ }^{1,2 *}$

\author{
AFFILIATIONS: \\ ${ }^{1}$ Department of Biological Sciences and Bioengineering, Indian Institute of Technology Kanpur, \\ Kanpur-208016, Uttar Pradesh, India and \\ ${ }^{2}$ The Mehta Family Centre for Engineering in Medicine, Indian Institute of Technology Kanpur, \\ Kanpur, Uttar Pradesh, India. \\ ${ }^{3}$ Arthritis \& Regenerative Medicine Laboratory, Institute of Medical Sciences, University of Aberdeen, \\ Aberdeen AB25 2ZD, UK \\ ${ }^{4}$ Department of Zoology, Banaras Hindu University, Varanasi-221005, Uttar Pradesh, India \\ \# These two authors contributed equally \\ ${ }^{*}$ Corresponding Author: Amitabha Bandyopadhyay \\ Email address: abandopa@iitk.ac.in
}

\section{Competing Interests:}

The authors declare the following competing interests:

The use of BMP inhibitors as locally administered agents using sustained drug delivery vehicle(s) has been submitted for patent via Indian patent application number - 201911044840.

The inventor(s) are:

1. Dr. Amitabha Bandyopadhyay-IIT Kanpur, India,

2. Mr. Akrit Pran Jaswal - IIT Kanpur, India.

3. Dr. Praveen Vemula - Institute for Stem Cell Biology and Regenerative Medicine, India.

4. Dr. Manohar Mahato - Institute for Stem Cell Biology and Regenerative Medicine, India.

CLASSIFICATION: Major: Biological Sciences Minor: Applied Biological Sciences

KEYWORDS: BMP, Osteoarthritis, articular cartilage, local inhibition, LDN-193189, post onset

AUTHOR CONTRIBUTIONS: A.B., A.P.J. designed the experiments and A.P.J., A.K.S., B.K., S.V.N. and S.F.I. conducted experiments, collected and analyzed data. A.P.J., A.K.S. and B.K. prepared the manuscript; N.A. conducted the cell-based LDN-193189 assay. A.B., C.D.B., A.J.R. edited the manuscript along with A.P.J.; A.K.S. provided the data for inflammation response studies and mechanistic data including Smurf expression analysis; A.J.R. and A.H.K.R. collected and analysed human cartilage samples; H.W. and S.A. performed the scoring for osteoarthritis. 


\section{ABSTRACT:}

Osteoarthritis $(\mathrm{OA})$ is a prominent musculoskeletal disorder with no effective therapy. Moreover, molecular aetiology of $\mathrm{OA}$ and the development and maintenance of articular cartilage are poorly understood. During OA, articular cartilage undergoes cellular and molecular changes reminiscent of transient cartilage, the embryonic precursor of endochondral bone. Previous studies from our lab suggest that during embryonic development, a precise spatio-temporally regulated WNT-BMP signaling interplay dictates differentiation of a common progenitor pool to either articular or transient cartilage fate in adjacent domains. While Wnt signaling promotes articular cartilage fate, transient cartilage differentiation is critically BMP signaling dependent. Moreover, any ectopic activation of BMP signalling embryonically, leads to ectopic transient cartilage differentiation at the expense of articular cartilage. In this study, we show that BMP signaling is sufficient and necessary for pathogenesis of OA by ectopically activating BMP signaling and depleting BMP ligands in adult mice articular cartilage, respectively. Similarly, human osteoarthritic specimens show upregulation of BMP signaling in the articular cartilage. A recent study based on our work (1), suggests that pharmacological inhibition of BMP signaling allows maintenance of hMSC derived chondrocytes, implanted in mice, for longer time duration (2). Similarly, we observe in vivo local pharmacological inhibition of BMP signaling resulted in delayed onset and reduced severity of $O A$ in a mouse model of $O A$ as well as long term protection from OA like changes. Therefore, pharmacological inhibition of BMP signaling and consequent block of transient cartilage differentiation of joint cartilage cells can be a potential disease modifying therapy for OA.

Significance statement: This manuscript provides new insights into the mechanistic basis for the maintenance of articular cartilage in adults. Here we demonstrate the role of BMP signaling in the pathogenesis of osteoarthritis in mice and humans. To the best of our understanding, this is the first (3)study investigating the relationship between molecular histological changes observed in the articular cartilage during osteoarthritis and inflammation. Moreover, we demonstrate that local inhibition of BMP signaling can be a potential disease modifying therapy for osteoarthritis which is among the most prominent musculoskeletal disorders.

\section{Introduction}

Osteoarthritis $(\mathrm{OA})$ is a painful, debilitating musculoskeletal disorder with profound socioeconomic burden and the primary cause of locomotive disability affecting millions of people worldwide (4-6). The alarmingly increasing prevalence of $O A$ is exacerbated further as no disease modifying therapy exists to manage $O A$ except for symptomatic treatment with anti-inflammatory drugs or surgical intervention in late stage disease (7). It is imperative therefore, to discern the molecular basis of pathogenesis of $\mathrm{OA}$ in order to develop a disease modifying therapy.

Articular cartilage, the tissue affected in OA, is a lubricated, avascular, alymphatic, aneural tissue that lines the ends of the bones at the joints. During OA, the joint surface undergoes a slew of changes characterised by loss of cartilage proteoglycans, hypertrophy of chondrocytes, angiogenesis, osteophyte formation, and ultimately failure of joint function (7). The cellular and molecular changes of the joint cartilage during the onset and progression of OA closely resemble the steps of endochondral ossification, the developmental process by which long bones form within cartilage anlagen.

During endochondral ossification, most of the initial cartilage mass in an appendicular skeletal element is replaced by newly forming bone except for the cartilage at the termini. The cartilage that is replaced by bone is referred to as the transient cartilage while the cartilage at the terminal ends is referred to as the joint or articular or permanent cartilage (8). During transient cartilage differentiation, type II collagen (Col2a1) expressing cartilage cells undergo a series of changes. These cells undergo pre-hypertrophic differentiation wherein they express Indian hedgehog $(\mathrm{IHH})$, subsequently the transition from pre-hypertrophy to hypertrophy is marked by the expression of type $\mathrm{X}$ collagen (ColX). The hypertrophic cells are infiltrated by blood vessels. This is followed by matrix remodelling, where enzymes viz. MMP-13 and ADAMTS-5, degrade the existing collagen matrix and a new matrix, rich in type I collagen (Coll), is synthesised and bone formation is accomplished $(9,10)$. Ray et.al. 
demonstrated that within a developing appendicular skeletal element there is a zone of Col2a1 expressing bipotential proliferating cell population referred to as the Distal Proliferative Zone (DPZ). The DPZ cells under the influence of BMP signaling undergo transient cartilage differentiation whereas when exposed to WNT signaling undergo joint cartilage differentiation (1). Some of the molecules involved in transient cartilage differentiation viz. MMP-13, ADAMTS-5, and VEGF-A are reported to be associated and/or necessary for the pathogenesis of OA (11-19).

Previous literature suggests that ectopic activation of BMP signaling in developing cartilage or presumptive joint site, either by overexpression of BMP ligands $(1,20)$ or misexpression of constitutively active BMP receptors (21), results in transient cartilage differentiation at the expense of joint cartilage. In addition, immobilisation of developing embryonic limbs leads to ectopic differentiation of transient cartilage at the cost of articular cartilage $(22,23)$. Moreover, it was shown that immobilization leads to ectopic upregulation of BMP signaling within the sub-articular cartilage domain where cartilage precursors are normally exposed only to WNT signaling $(22,24)$. Similarly, in a recent study,it was also demonstrated that pharmacological inhibition of BMP signalling promotes articular cartilage differentiation in hMSC derived chondrocytes and allowed the cells to maintain articular chondrocyte phenotype for longer duration of time upon implantation in mice (2), suggesting that embryonic paradigm of spatial restriction of BMP signalling is needed for differentiation and maintenance of articular cartilage phenotype.

Taken together, we hypothesize that BMP signaling induced transient cartilage differentiation within adult articular cartilage domain is the molecular basis of the pathogenesis of OA.In this manuscript we tested this hypothesis with conditional gain- and loss-of-function mouse mutants of BMP signaling in conjunction with a surgically induced model of OA. Our study in mice is also supported by data obtained from osteoarthritic human cartilage specimens, wherein we found evidence of active BMP signaling in the joint cartilage. Further, our data suggests that in situ pharmacological inhibition of BMP signaling in the synovial joint may serve as an effective disease modifying therapy for OA.

\section{RESULTS:}

\section{Ectopic activation of BMP signaling in adult joint cartilage is sufficient to induce the development of an OA-like phenotype in mice}

To examine whether ectopic activation of BMP signaling in the articular cartilage is sufficient to induce osteoarthritic changes in adult mouse we generated pMes-caBmpr1a; Col2a1-Cre-ERT2 mouse strain, where BMP signaling can be ectopically activated in adult articular cartilage upon administration of tamoxifen (Fig.1A). We activated BMP signaling in postnatal cartilage at P56 by injecting tamoxifen intraperitoneally (referred to as induction from hereon) to pMes-caBmpr1a; Col2a1-Cre-ERT2 mice. Adult mice articular cartilage is devoid of active BMP signaling (Fig.1B). However, within seven days of mis-expression of caBmpr1a in adult mouse cartilage ectopic activation of canonical BMP signaling, as assessed by immunoreactivity towards phosphorylated SMAD1/5/8 (pSMAD1/5/8), is observed and it peaks after two weeks (Fig.1B'-B"'). Subsequently, markers of transient cartilage differentiation were also observed in the articular cartilage domain in the same sequence as observed during endochondral ossification. Expression of $\mathrm{IHH}$, which marks prehypertrophic state of cartilage, was observed within 7 days of induction and concurrent with the first detection of pSMAD1/5/8 immunoreactivity. IHH expression is downregulated by the $14^{\text {th }}$ day after induction (Fig.1C-C").ColX expression, which marks cartilage hypertrophy, was observed 14 days post induction and the maximum extent of hypertrophy was at 28 days post induction (Fig. 1D-D"'). Embryonic $(1,25)$ as well as adult articular cartilage cells $(2)$ are proliferation deficient while transient cartilage cells are proliferative (1). In our experiments we observed that along with other makers of transient cartilage differentiation, proliferation was also stimulated in the adult mice articular cartilage upon induction. Increased BrdU uptake was observed in the adult joint cartilage 7 days after induction which was more apparent on the $14^{\text {th }}$ day post induction (Fig.1E-E"). 
At 60 days post induction, an upregulation of matrix remodelling enzyme MMP-13 was also observed in articular cartilage cells (Fig.1F-F'). Moreover, Coll, marking osteogenic differentiation, was also found to be expressed in multiple zones of articular cartilage 60 days post activation of BMP signaling (Fig.1G-G'). Six months post induction, widespread upregulation of MMP-13 and Coll was observed throughout all zones of articular cartilage suggesting terminal osteogenic differentiation had occurred (Fig. 1F" and Fig. 1G"). Focal loss of proteoglycan staining in multiple zones of articular cartilage was also observed (Fig. 1H-1H').

Further, we observed the appearance of NITEGE immunoreactivity in the adult articular cartilage 6 months post induction (Fig. 1l-1/'). Besides the molecular signatures, the most conspicuous feature of osteoarthritic articular cartilage is the formation of osteophytes in the joint, which is otherwise devoid of such features. Micro CT imaging of hind limbs revealed extensive osteophyte (indicated by black arrow) formation upon ectopic activation of Bmpr1a in articular cartilage (Fig.1J). Taken together, these observations strongly suggest that ectopic activation of BMP signaling is sufficient to induce the development of an OA like phenotype in adult mice.

\section{BMP signaling induced transient cartilage differentiation is necessary for the pathogenesis of $O A$}

Next, we investigated the necessity of BMP signaling in the development of osteoarthritic phenotype. It has been previously reported that levels of BMP-2 ligands are elevated in synovial fluid from OA patients and BMP receptor localisation is associated with OA severity $(26,27)$. However, the presence of active BMP signaling in situ osteoarthritic cartilage has not been reported. To induce OA

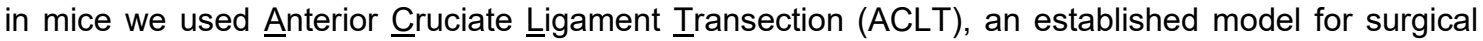
induction of experimental OA $(28,29)$. We chose to analyse the knee articular cartilage every week after ACLT for the appearance of active BMP signaling. As opposed to sham operated knees (Fig. S1A) or 7 days post ACLT (Fig. S1B) we observed increased pSMAD1/5/8 immunoreactivity in specimens from 14 days post ACLT (Fig. S1B') which persisted till 56 days post-ACLT (Fig. S1B", Fig. $\mathrm{S}^{\prime} \mathrm{B}^{\prime \prime \prime}$ and Fig. 2B'). In keeping with our observation in the context of ectopic activation of BMP signaling, we observed increased uptake of BrdU in the articular cartilage of mice post ACLT (Fig. S1D-D"') in contrast to the sham-operated group (Fig. S1C). In order to prevent activation of BMP signaling post ACLT, we used a previously described Bmp2/4 double conditional knockout strain (30). We crossed this strain with Col2a1-Cre-ERT2 to create Bmp2/c; Bmp4c/c; Col2a1-Cre-ERT2 strain. This allowed us to deplete BMP ligands in the articular cartilage in a temporally controlled manner through tamoxifen injection. We injected tamoxifen intraperitoneally in P56 mice. ACLT was performed at P120 (Fig. S2A and Fig. 2A).

As expected, we could not detect any upregulation of BMP signaling in the articular cartilage of Bmp2/4 depleted animals when subjected to ACLT (Fig. S2B" and Fig. 2B"). Distribution and abundance of Colll was found to be normal in Bmp2/4 depleted animals even after 56 days of ACLT (Fig. S2C-C" and Fig. 2C-C"). Cartilage hypertrophy, as assessed by ColX immunoreactivity (Fig. 2D$\left.\mathrm{D}^{\prime \prime}\right)$ as well as expression of MMP-13 (Fig. 2E-E"), a key matrix remodelling enzyme, were significantly elevated after 56 days of ACLT (Fig. 2D' and Fig. 2E'). However, depletion of Bmp2/4 protected against extensive upregulation and allowed the maintenance of a level of ColX (Fig. 2D") and MMP-13 (Fig. 2E") almost comparable to that of sham (Fig. 2D and Fig. 2E). We also noted that while cartilage loss of proteoglycan staining was observed in ACLT specimens, these changes were minimum post depletion of BMP ligands (Fig. 2F-F"). The emergence of neo-epitope NITEGE resulting from aggrecan degradation was also considerably reduced in Bmp2/4 depleted articular cartilage (compare, Fig. 2G" with Fig. 2G'). Similar observations were made 28 days post ACLT as well (Fig. S2G-S2G"). Moreover, Coll expression, which is a marker for bone differentiation (or ossification), was also found to be at levels significantly lower in articular cartilage post ACLT in BMP depleted tissues as compared to vehicle injected ACLT control animals(compare, Fig. $2 \mathrm{H}^{\prime \prime}$ with Fig. $\left.2 \mathrm{H}^{\prime}\right)$. Structural examination using micro-computed tomography $(\mu \mathrm{CT})$ revealed that in "ACLT + Vehicle" group, extensive damage to articular surfaces (roughness) as well as osteophyte (marked by red arrows) formation was observed (Fig.2l'). However, upon depletion of BMP ligands, the severity and extent of these changes were significantly attenuated (Fig. 2l"), and cartilage quality 
(smoothness) in this group appeared comparable to that in sham operated group (Fig.2I) suggesting cartilage protection was afforded.

To ascertain the clinical relevance of these findings we examined both osteoarthritic and nonosteoarthritic human articular cartilage. Immunoreactivity of pSMAD1/5/8 was observed in all zones of osteoarthritic cartilage from patients who underwent arthroplasty (Fig.2J", $2 \mathrm{~J}$ '"), while human cartilage from a donor without known history of OA showed minimal detectable pSMAD1/5/8 immunoreactivity (Fig. 2K", 2K"'). There was no pSMAD1/5/8 immunoreactivity in osteoarthritic cartilage treated with phosphatase (Fig.2J', 2K').

\section{Local pharmacological inhibition of BMP signaling halts the progression of osteoarthritic changes}

We next investigated whether inhibiting BMP signaling locally in articular joints can retard the progress of osteoarthritic changes after surgical induction of OA. For this purpose we used a well characterized BMP signaling inhibitor, LDN-193189, a derivative of dorsomorphin (31-33). LDN193189 activity was assayed using BRITER (BMP Responsive Immortalized Reporter) cell line (34) (please see Materials and Methods). As low as 100nM concentration of LDN-193189 significantly inhibited BMP signaling in BRITER cell line (Fig. S3).

Considering possible dilution and volume loss of LDN-19389 during the injection, we used $10 \mu \mathrm{M}$ (in $3 \%$ w/v 2-hydroxypropyl- $\beta$-cyclodextrin in PBS) of LDN-193189 for intra-articular injection to locally inhibit BMP signaling. We devised a regime where we performed ACLT on wild type mice at P120 and injected LDN-193189 into the knee joint intra-articular space for seven consecutive days starting on the 14th day post-surgery and harvested 28 days post-surgery (Fig.3A).

We observed that local inhibition of BMP signaling severely abrogates OA-associated changes in articular cartilage post transection of ACL. No immunoreactivity to pSMAD1/5/8 was observed in articular cartilage of LDN-treated set post ACLT (Fig. 3B") and the sham operated knee cartilage (Fig.3B), while pSMAD1/5/8 positive cells were found in zones of articular cartilage in vehicle administered ACLT knee joints (Fig.3B'). The level and distribution of Colll was native like in LDN193189 treated knee joints (Fig.3C") as compared to ACLT control set (Fig.3C') suggesting reduced depletion of Colll post-surgery and protection of cartilage. Hypertrophy of cartilage cells was found to be restricted to the calcified zones and minimal ColX immunostaining was observed in the articular cartilage of LDN-treated ACLT knees (Fig. 3D") similar to sham set (Fig.3D), while vehicle injected animals showed extensive hypertrophy throughout the cartilage matrix (Fig.3D'). Similarly, MMP-13 levels were found to be significantly reduced in articular cartilage upon intra-articular administration of LDN-193189 (Fig.3E") whereas in vehicle injected knee joints, a global upregulation of MMP-13 was observed throughout multiple zones of articular cartilage (Fig.3E'). Further, we observed that articular cartilage in LDN-193189 treated group was devoid of any immunoreactivity to NITEGE (Fig.3F") similar to what was observed in the sham set (Fig.3F), suggesting lesser aggrecanolysis by aggrecanolytic enzymes while abundant NITEGE expression was observed in vehicle treated specimens (Fig. $\left.3 F^{\prime}\right)$. Proteoglycan depletion and cartilage damage was observed to be minimal in tibial surface of LDN-193189 injected (Fig.3G") as compared to vehicle injected controls (Fig. 3G') and cartilage integrity was found to be comparable to sham operated knees (Fig. 3G). To examine the status of terminal transient cartilage differentiation following completion of LDN-193189 regime, we carried out immunostaining for Coll. As opposed to vehicle treated ACLT group (Fig.3H'), we found that very few cells in articular cartilage of LDN-193189 treated joints expressed Coll (Fig.3H"). The structural parameters were examined by $\mu \mathrm{CT}$ imaging. We observed that articular cartilage quality was maintained in terms of minimal erosion, osteophytes formation and less pronounced surface changes upon local inhibition of BMP signaling by using LDN-193189 intra-articular injection (Fig. 3II", red arrows mark osteophytes). Moreover, OARSI scoring of cartilage revealed a significantly attenuated osteoarthritic-like phenotype in LDN-193189 treated group as compared to the vehicletreated ACLT group (Fig.3J). 
Taken together, these findings suggest that in situ inhibition of BMP signaling in articular cartilage is sufficient to prevent the phenotypic and molecular changes associated with development and progression of OA in a surgically induced osteoarthritic mouse model.

\section{Inhibition of BMP signaling post onset of OA attenuates disease severity}

In situ inhibition of BMP signaling before the onset of OA following ACL transection in mice retards the progression of OA. However, in a clinical setting, patients report to the clinic after the disease has set in. We therefore investigated if local inhibition of BMP signaling can mitigate the severity of osteoarthritic changes even after the disease has set in. For this purpose, seven consecutive intraarticular LDN-193189 injections were administered starting on post-surgery day 35 and finishing on post-surgery day 42 . The knees were harvested at post-surgery day 56 (Fig.4A).

In contrast to vehicle treated knee joints, where ColX positive cells could be observed throughout articular cartilage (Fig.4B') in LDN-193189 treated samples, hypertrophic cells were found restricted only to a few upper zone cells of articular cartilage (Fig. 4B"). The level of MMP-13 was also observed to be markedly less in the articular cartilage of LDN-193189 administered knees (Fig.4C"), as compared to the vehicle control ACLT group (Fig.4C') where a high level of MMP-13 expression was observed throughout. Moreover, NITEGE neo-epitope was absent from the middle or deeper zones of articular cartilage in LDN-193189 administered knees (Fig.4D") as opposed to vehicle treated ACLT set (Fig.4D') where such cells were found throughout the depth of articular cartilage. Proteoglycan staining was found to be more intense in certain areas of LDN-193189 treated knee joints (Fig.4E") which did not coincide with any known marker of hypertrophy or transient cartilage differentiation while overt signs of OA were observed in vehicle treated ACLT knee cartilage (Fig.4E'). Similarly, while Coll positive cells were found in vehicle treated ACLT set (Fig.4F'), such cells were not observed in the LDN-193189 treated knee specimens (Fig.4F") or in the sham operated group (Fig.4F). Evaluation of the structural parameters using $\mu \mathrm{CT}$ imaging revealed that cartilage surface erosion was reduced in LDN-193189 treated set as compared to vehicle injected knees (Fig.4G-4G", red arrow marks osteophytes). The OARSI scores in LDN-193189-treated group were significantly lower than that for ACLT group, even though administration of LDN-193189 was performed after the onset of disease (Fig. $4 \mathrm{H}$ ). It should be noted though that less protection of cartilage was afforded, as judged by the OARSI severity scores, to the knee joints treated with LDN-193189 post-onset of OA compared to when knee joints were treated with LDN-193189 pre-onset of OA (compare Fig. 3J and Fig. $4 \mathrm{H})$.

We have observed that intra-articular administration of LDN-193189 provides protection against OA like changes at least for 14 days post injection (Fig. 4). Next, we wanted to investigate the potential for clinical translatability of LDN-193189 or similar molecule as a disease modifying agent. We examined whether LDN-193189 can confer longer term protection against surgically induced OA by emulating a clinic-like regimen of minimum dosage and maximum efficacy over extended durations of time. Our data (Fig. S2) as well as existing literature (35) suggest that molecular changes associated with OA are apparent within 28 days of ACLT. Hence, we conducted ACLT at P120, injected LDN193189 intra-articularly on PS28, PS30 and PS32 and harvested the knee joint 56 days later at PS84. As compared to vehicle control, in the LDN-193189 injected specimen, Colll expression (compare Fig.5B with Fig.5B"') as well as cartilage specific proteoglycan content, as assessed by Safranin O staining (compare Fig.5D with Fig.5D'"), were largely preserved. Also, ColX immunoreactivity was significantly lower in LDN-193189 treated specimen (compare Fig.5C with Fig.5C'"'). This set of data suggests that even after onset of surgically induced OA, blocking BMP signaling pathway locally can offer protection for at least 56 days in mice.

\section{Mechanistic insight into the pathogenesis of OA from a developmental biology perspective}

Recently, Singh et al., demonstrated that immobilisation of chick or mouse embryos results in transient cartilage differentiation at the expense of articular cartilage differentiation which is associated with ectopic activation of BMP signaling (22). Further, this study also demonstrated that this ectopic activation is associated with a concurrent downregulation of expression of SMURF1, an 
intracellular inhibitor of BMP signaling pathway (22). We detected a downregulation of SMURF1 (Fig.5E-E") expression in the mouse articular cartilage 30 or 60 days post ACLT. This suggests that the molecular mechanism of articular cartilage maintenance via mechanical regulation is conserved between embryonic and postnatal stages and likely involved in pathologies such as OA.

\section{Effect of local inhibition of BMP signaling on inflammatory responses in a surgically induced osteoarthritic mouse model}

We performed an analysis for candidate inflammatory response molecules which are known to be involved in the development of osteoarthritis $(36,37)$. IL-1 $\beta$ immunostaining showed that while there was an increased immunoreactivity to IL-1 $\beta$ in the vehicle-treated group (Fig.6 $\mathrm{A}^{\prime}$ ), minimal reactivity could be detected in LDN-193189 treated mice (Fig.6A"),comparable to that observed in the shamoperated group (Fig.6A). Similarly, we performed immunostaining for iNOS, which is a known key player in the development of OA. iNOS was detected in the subchondral bone of sham-operated mice (Fig.6B) and upon ACLT, iNOS was also detected in some of the articular cartilage cells of vehicle treated mice (Fig.6B') while no such cell could be detected in the LDN-193189 treated group (Fig.6B"). Immunoreactivity for NF-kB was detected in the articular cartilage of the vehicle treated ACLT group (Fig.6C'), while it could not be detected in either the LDN-193189 treated group or in the sham operated group (Fig.6C" and 6C, respectively). We also investigated TNF- $\alpha$ immunoreactivity in osteoarthritic cartilage upon treatment with LDN-193189 and found that TNF- $\alpha$ immunoreactivity was observed in the ACLT group injected with vehicle (Fig.6D', white arrow), while the LDN-193189 treated group displayed minimal immunoreactivity (Fig.6D"), similar to that in sham-operated mice where TNF- $\alpha$ could be detected in subchondral bone (Fig.6D).

\section{Discussion:}

Our findings show that there exists striking resemblance between the molecular changes associated with the onset of osteoarthritic changes in the articular cartilage and the molecular signature associated with endochondral ossification. Moreover, the temporal order of the expression of molecular markers post ACLT induced OA was found to be strikingly similar to what is observed with transient cartilage differentiation during endochondral ossification. Inhibition of transient cartilage differentiation via blocking $\mathrm{IHH}$ signaling has been reported to inhibit and attenuate the severity of osteoarthritic phenotype post ACLT(38-40). However, so far, no IHH signaling inhibitor has been approved for clinical use. This could be because Ihh loss-of-function, during embryonic development, results only in a temporary delay in hypertrophic differentiation - an essential step of endochondral ossification, which eventually gets restored and even accelerated at postnatal stages in mice $(41,42)$. Taken together, these studies give a crucial hint that blocking transient cartilage differentiation is a viable strategy to manage osteoarthritic changes in the articular cartilage. This hypothesis is in line with what has been suggested earlier in the literature (43-45).

BMP signaling is known to play a critical role in transient cartilage differentiation. Previously, intraperitoneal administration of a BMP signaling inhibitor, LDN-193189, has been shown to reverse the phenotype associated with Fibrodysplasiaossificans progressiva, a condition where progressive heterotopic ossification of muscle is observed upon injury, due to constitutive activation of BMP signaling (31). In this study, we found that activation of BMP signaling is both necessary and sufficient for the pathogenesis of OA in mice. The necessity of BMP signaling in the onset of osteoarthritis like changes in the articular cartilage has been demonstrated using both genetic and pharmacological means whereas sufficiency has been demonstrated using genetic means. Further, analysis of patient samples suggests an association between osteoarthritis and activation of BMP signaling in the articular cartilage cells. Since we have used Col2a1-Cre-ERT2 mediated recombination as the means to activate expression of caBMPRIA we cannot rule out the possibility that BMP signaling has also been activated in the growth plate cartilage of adult mice and the molecular and cellular changes observed is partly due to activated BMP signaling in the growth plate cartilage. However, in all our analyses (Fig. 1) the changes were first observed in the superficial layers of articular cartilage 
suggesting that the changes observed were primarily due to ectopic activation of BMP signaling in the articular cartilage.

Interestingly, we also observed a surge in proliferation in articular cartilage cells, as assessed by enhanced BrdU uptake, post ACLT or activation of BMP signaling. Our data suggests that articular cartilage cells, originally having low regenerative potential and proliferative capacity, display a regenerative response upon ACLT or upregulation of BMP signaling. However, this leads to an altered tissue microenvironment that promotes transient cartilage differentiation at the expense of articular cartilage. Thus, instead of healing by regeneration it further promotes the disease condition. Prophylactically blocking BMP signaling in situ using LDN-193189 led to an attenuation in the severity of osteoarthritic phenotype following surgical induction in mice. Further, our investigation suggests that administration of LDN-193189 after the onset of OA not only halts the progression of OA but also an intense toluidine blue stained cartilage tissue appears which is negative for transient cartilage markers, suggesting that new cartilage formation takes place.

Finally, it has to be acknowledged that while transient cartilage differentiation could be involved in the initiation of the disease, the inflammation associated with $O A$ can determine the severity and course of disease progression $(46,47)$. However, despite a large body of existing literature exploring the role of inflammation in the pathology of $\mathrm{OA}$, there is no clear demonstration of the hierarchy between onset of inflammation and transient cartilage differentiation. Our data suggest that pharmacologically blocking BMP signaling in surgically induced OA knees prevents activation of inflammatory response as well (Fig. 6). Our data suggest that in situ inhibition of BMP signaling,and consequently transient cartilage differentiation, may be a potent means of disease modifying therapy for osteoarthritis.

\section{Materials and Methods}

\section{Study design:}

Sample size: We used statistical power calculations using $\mathrm{G}^{*}$ Power3.1 software to generate the sample size from previously conducted studies in mice using Bmp2/4 DCKO mice $(25,48)$.

Data Exclusion: No data was excluded from the end study.

Rules for stopping data collection: Data collection was stopped at the end points pertinent to different experiments since tissues were harvested at the end of each experiment. The relevant time points were determined for each experiment based on similar experiments reported previously as well as from developmental biology insights and no outliers were present or reported(35).

Replication of data was ensured for all experiments including protocols involving classical histology and molecular immunohistochemistry by repeating at least three times to ensure reproducibility of data.

Experimental design: The present study is a controlled laboratory experiment involving animal subject, human specimens, and cell culture studies. Primary nature of data collected, recorded, and analyzed was imaging based data, viz. microscopic imaging, microCT imaging data and statistical softwares used accordingly.

Randomization: Age, gender and genotype matched animals were used to minimize randomization in experiments. For all the genetic induction experiments 2 groups were used: 1) Vehicle injected and 2) tamoxifen injected. In experiments involving surgical induction with or without genetic induction, 3 groups were used: 1) Sham surgical control 2) ACLT + vehicle 3) ACLT + Tamoxifen / LDN-193189 treated, depending on the type of experiment.

Blinding: OARSI scoring was performed by the researchers who were not involved in the animal experiments and who were blinded to the treatment groups. Data was then unblinded and analysed by the researchers carrying out the experiments.

Details for the animal and human subjects are provided in the following sections. 


\section{Generation of mice lines}

All animals were housed, bred and maintained in Central Experimental Animal Facility (CEAF) of Indian Institute of Technology Kanpur, India. All experiments were performed in accordance with the guidelines of the Institutional Animal Ethics Committee (IAEC) as well as under the aegis of the Centre for Purpose of Control and Supervision of Experiments on Animals (CPCSEA), Government of India under protocols IITK/IAEC/2013/1002; IITK/IAEC/2013/1015; and IITK/IAEC/2013/1040. We obtained B6By/J wild type mice, TgCol2a1-Cre-ERT2(48) and ROSA26 mT/mG(49)strainsfrom Jackson Laboratories, USA; pMes-caBmpr1a mice as gift from Prof. YiPing Chen at Tulane University, USA; Bmp2c/; $B m p 4^{c / c}$ mice from Prof. Clifford Tabin at Harvard Medical School, USA. For BMP signaling gain-of-function, pMes-caBmpr1a mice were crossed with TgCol2a1-Cre-ERT2 mice to generate pMes-caBmpr1a; TgCol2a1-Cre-ERT2. Bmp2 ${ }^{c / c} ; B m p 4^{c / c}$ (30)animals were crossed with TgCol2a1-Cre-ERT2 to generate $B m p 2^{c / c}$; Bmp4c/c; TgCol2a1-Cre-ERT2 for BMP loss-of-function mutation. Mice were genotyped for the presence of caBmpr1a using PCR with primers $5^{\prime}$ CGAAGATATGCGTGAGGTTGTGT-3' and 5'- TGgGCGAGAGgGgAAAGAC- 3' while TgCol2a1Cre-ERT2 transgene was genotyped using primer pair 5'- CACTGCGGGCTCTACTTCAT-3' and 5'ACCAGCAGCACTTTTGGAAG- $3^{\prime}$. ROSA26 $\mathrm{mT} / \mathrm{mG}$ was genotyped using primers 5'CTCTGCTGCCTCCTGGCTTCT-3' and 5'- TCAATG GGCGGGGGTCGTT - 3'. Bmp2/cwas genotyped using 5'- GTGTGGTCCACCGCATCAC-3' and 5'- GGCAGACATTGTATCTCTAGG- 3' and $B m p 4^{c / c}$ was genotyped using 5'-AGACTCTTTAGTGAGCATTTTCAAC-3' and 5'AGCCCAATTTCCACAACTTC- 3' primer pairs.

\section{Anterior Cruciate Ligament Transection (ACLT)}

All ACLT surgeries were conducted at P120. Unless specifically mentioned, ACLT was conducted on $B 6 B y / J$ wild type male mice.Animals were subjected to ACLT at P120 and harvested at post ACLT day $07,14,21,28$, and 56 for molecular and histological analysis. SMURF1 immunoreactivity was analyzed after one and two months of ACLT in wild type mice. All animals were anesthetized using isoflurane during ACLT surgery following standard protocol (29).ACLT was performed in left hind limb of all mice.Patella was dislocated medially to expose femorotibial joint cavity and ACL was transected using a micro-surgical blade under the microscope. After ACL transection, patella was repositioned and quadriceps femoris muscle was sutured to prevent its dislocation. In the sham group, the joint cavity and ACL were exposed but left intact and sutured. Surgical incision was sealed using Vicryl 5-0 Absorbable suture. Povidone-lodine solution was applied over the sutured skin to prevent microbial infections.

\section{BMP signaling gain-of-function}

pMes-caBmpr1a;TgCol2a1-Cre-ERT2 male mice were injected with three consecutive doses of tamoxifen (Sigma-Aldrich, $2.5 \mathrm{mg} / 25 \mathrm{~g}$ body weight) starting from P56 to induce Cre mediated 
recombination and harvested at post TAM injection day 7,14, 21, 28, 56 and 6 months for molecular and histological analysis.

\section{BMP signaling loss-of-function}

All animals used for BMP signaling loss-of-function were homozygous males for the conditional alleles of Bmp2 and Bmp4. Three doses of tamoxifen (Sigma-Aldrich, $2.5 \mathrm{mg} / 25 \mathrm{~g}$ body weight) were injected in $\mathrm{Bmp2}^{\mathrm{c} / \mathrm{c}} ; \mathrm{Bmp} 4^{\mathrm{c} / \mathrm{c}}$; TgCol2a1-Cre-ERT2 miceintraperitoneally starting from P56 for three consecutive days to activate Cre-mediated recombination to deplete BMP2 and BMP4 in adult cartilage. pSMAD1/5/8 immunoreactivity was used to assess the status of BMP signaling before/after ACLT and/or with/without TAM-induced recombination.

Local inhibition of BMP signaling was achieved through administration of $6 \mu \mathrm{l}$ solution of $10 \mu \mathrm{M}$ LDN193189, prepared in 3\% 2-(hydroxypropyl)- $\beta$-cyclodextrin (w/v in PBS) in the knee joint cavity. Local inhibition of BMP signaling was done at two different time points. In one set, LDN-193189 was injected daily from $14^{\text {th }}$ to $21^{\text {st }}$ day post ACLT. In the other set LDN-193189 was administered daily from $35^{\text {th }}$ to $42^{\text {nd }}$ day post ACLT. For the experiment conducted to assess the dosage required and the duration of protection offered by LDN-193189 ACLT was conducted on P120 and LDN-193189 was injected

To observe the persistent effect of LDN-193189 over a prolonged period as well as to ascertain the optimum number of doses needed for providing long term protection following ACLT, LDN-193189 was injected on post-surgery day 28 (single dose) or post-surgery days 28 and 30 (two doses) or post-surgery day 28,30 and 32 (three doses). Tissues were harvested at post ACLT day 84 for further analysis. In all experiments the vehicle control animals were injected with 3\% 2-(hydroxypropyl)- $\beta$ cyclodextrin ( $w / v$ in PBS).

\section{Cell proliferation analysis}

Cell proliferation status was assessed using BrdU incorporation assay following intraperitoneal delivery of $100 \mathrm{mg} \mathrm{BrdU} / 10 \mathrm{ml} \mathrm{PBS} / \mathrm{kg}$ of body weight for three consecutive days prior to the day of harvesting tissues.

\section{LDN-193189 activity assay}

BRITER (BMP Responsive Immortalized Reporter) cell line (34) were used to determine optimum concentration of LDN-193189 required to inhibit BMP signaling.

\section{Human sample collection}

Osteoarthritic cartilage from 6 patients and non-osteoarthritic cartilage from one patient undergoing knee joint excision for malignancy at a site not involving sampled area, were obtained after informed consent and in accordance with the relevant guidelines and regulations, with approval from the NHS Grampian Biorepository Tissue Bank Committee, UK.

\section{Processing of tissue, histology and immunohistochemistry}


Post harvesting, tissues were fixed in $4 \%$ paraformaldehyde for $16 \mathrm{~h}$ at $4{ }^{\circ} \mathrm{C}$ and then washed in PBS for $2 \mathrm{~h}$. The tissues were decalcified in $14 \%$ EDTA solution $(\mathrm{pH} 7.4)$ for 28 days with regular changes of EDTA solution at least once in a week. Decalcified tissues were embedded in paraffin wax and sectioned at $5 \mu \mathrm{m}$ thickness.

For immunohistochemistry, sections were treated with xylene and then rehydrated using descending alcohol gradient. Antigen retrieval was done using citrate buffer except for collagens. Coll, Colll and ColX antigens were retrieved by treatment of acidic solution of $0.05 \%$ pepsin at $37^{\circ} \mathrm{C}$ for 25 minutes. Tissue sections were washed with PBS and treated with $3 \% \mathrm{H}_{2} \mathrm{O}_{2}$ for 15 minutes to block endogenous peroxidase activity. After blocking in 5\% HINGS (Heat-inactivated Goat Serum) solution for an hour, tissue sections were incubated with primary antibodies against Type $X$ Collagen (DSHB, Cat.no. X-AC9-s, 1:20), Type II Collagen (DSHB, Cat.no. CIIC1-s, 1:20), NITEGE (Abcam, Cat.no. ab3773,1:20), SMURF1 (Santa Cruz Biotechnology,Cat.no.SC100616,1:100), NF-kB (CST,Cat.no.D14E12,1:400), IL-1ß (CST, Cat.no.3A6,1:100), MMP-13 (Santa Cruz Biotechnology, Cat.no.SC30073,1:100), pSMAD1/5/8 (CST Cat.no.13820S, 1:100), GFP (Invitrogen, Cat no.A6455, 1:100), BrdU (Sigma, Cat.no. B8434), iNOS (Abcam, Cat. no.3523, 1:50), and TNF-a (Santa Cruz Biotechnology, Cat.no. SC-52746, 1:100) for 16 hours at $4^{\circ} \mathrm{C}$. Tissue sections were washed with PBST and incubated with fluorescence labelled secondary antibodies for overnight at $4^{\circ} \mathrm{C}$. Secondary antibody against BrdU was conjugated with HRP (horseradish peroxidase) and detected by DAB (3,3 Diaminobenzidine).

Histological analysis was performed using Safranin $\mathrm{O}$ and Toluidine blue staining following standard protocols. For Safranin O/ Fast green staining, rehydrated tissue sections were treated with haematoxylin solution for 10 minutes, washed in running tap water, and treated with $0.5 \%$ Fast Green solution for 2 minutes. Thereafter, sections were dipped in 1\% acetic acid and finally stained with $0.5 \%$ Safranin O solution for 2 minutes. Stained sections were dehydrated with $95 \%$ and $100 \%$ alcohol respectively, cleared with Xylene and mounted in DPX. Toluidine blue staining was performed on rehydrated tissue sections with $0.05 \%$ toluidine blue solution and washed in tap water. Slides were air dried, treated with xylene and mounted in DPX.

Human cartilage sections were stained by immunohistochemistry for $\mathrm{pSmad} 1 / 5 / 8$ using standard protocols (3). Briefly, antigen retrieval was performed by incubation for 1 hour at $65^{\circ} \mathrm{C}$ in antigen unmasking citrate buffer solution ( $\mathrm{pH} \mathrm{6}$, Vector Laboratories, UK), after which sections were incubated with phosphatase (CST Lambda Phosphatase kit, New England Biolabs, \#P0753) according to manufacturer's instructions, or incubated with buffer only. Sections were then incubated with an antibody against pSmad1/5/8 (Millipore, cat no. ab3848, at 1:50 $(10 \mu \mathrm{g} / \mathrm{ml}$ )) followed by biotinylated goat anti-rabbit IgG (Vector Labs, cat no. BA-1000, at 1:200). Stained sections were imaged using Zeiss Axioskop 40 (Zeiss) with Progress XT Core 5 colour digital camera and ProgResCapturePro 2.9.0.1 software (JenOptik, Germany).

The OARSI scores were calculated using the recommended guidelines for assessment of osteoarthritic severity in small animals (mice). OARSI scoring was performed by researchers who were not involved in the animal experiments and who were blinded to the treatment groups. Data was 
then unblinded and analysed by the researchers carrying out the experiments. All images were taken using Leica DM 5000B compound microscope and processed using manufacturer provided software suite(s).

\section{Micro-Computed Tomography $(\mu \mathrm{CT})$}

Fixed tissue were taken in $5 \mathrm{ml}$ microfuge tube in hydrated condition and imaged using high resolution $\mu C T$ (Skyscan 1172). Images were reconstructed and analyzed using NRecon v1.6 and CTAn 1.16.8.0, respectively. 3-D volumetric projections were generated using CTVol 2.0 software. The scanner was set to operate at a voltage of $50 \mathrm{kVp}$, a current of $200 \mu \mathrm{A}$ and the resolution of the images was $5.86 \mu \mathrm{m}$ per pixel. $\mu \mathrm{CT}$ for BMP signaling gain-of-function specimens was performed, processed and analyzed at the Institutional facility of Western University, Ontario, Canadausing eXplore Locus GE scanner. The resolution of the image is $40 \mu \mathrm{m}$ per pixel and 3-D isosurface projection was generated using Parallax Innovations MicroView 2.5.0- rc15 (2.5.0-3557).

\section{Statistics}

Graph Pad Prism 8 software was used to ascertain statistical significance of the OARSI histopathological scoring data. One-way ANOVA with post-hoc analysis (Dunnett's test) was performed to calculate the statistical significance and interdependence between different experimental groups. The results were plotted using Graph Pad Prism 8 software. The error bars represent Standard Deviation (S.D.).

\section{Acknowledgements}

We are immensely grateful to Prof. YiPing Chen at Tulane University, USA for the gift of mouse strains. We thank Prof. Frank Beier of Western University, Ontario, Canada for teaching APJ the method of ACL transection. We sincerely thank Shuchi Arora and Ankita Jena for their critical comments on the manuscript and Niveda Udaykumar for help with statistical analysis. We thank Mr. Naresh Gupta for assistance with mouse experiments.

\section{Funding}

This work was supported by grants from the Department of Biotechnology, India (DBT) BT/PR17362/MED/30/1648/2017 and BT/IN/DENMARK/08/JD/2016 to A.B.; Versus Arthritis Grants 19667 and 21156 to CDB and AJR, Fellowships to APJ, BK, and SFI are supported by fellowships from the Ministry of Education, Govt. of India. Fellowship to AKS was supported by Science and Engineering Research Board, Govt. of India. APJ travelled to Western University Canada with Shastri Research Student Fellowship (SRSF, 2015-'16). A.H.K.R. was supported by the Wellcome Trust through the Scottish Translational Medicine and Therapeutics Initiative (Grant No. WT 085664).

\section{Legends to Figures}

Fig. 1. Ectopic activation of BMP signaling in adult joint cartilage is sufficient to induce OA development. 
(A)Schematic for generation of pMes-caBmpr1a; Col2a1-Cre-ERT2 mice and mis-expression of constitutively active Bmpr1a in the adult cartilage by injecting tamoxifen (TAM) intraperitoneally at P56. (B-D"') Longitudinal sections through the adult knee joints of vehicle control (B-D)and mice 7 days ( $\left.B^{\prime}-D^{\prime}\right), 14$ days $\left(B^{\prime \prime}-D^{\prime \prime}\right), 28$ days ( $\left.B^{\prime \prime \prime}, D^{\prime \prime \prime}\right)$ post induction by TAM injection. Immunoreactivity for pSMAD1/5/8 (B-B"'), IHH (C-C") and ColX (D-D"'). (E-E")BrdU incorporation after 7 days (E') and 14 days $(E ")$ of TAM injection. (E) Vehicle control. Insets display the higher magnification view of the marked regions in (E-E"). (F-G") Immunoreactivity for MMP-13 (F-F") and Coll (G-G") in vehicle control $(F, G)$ and TAM injected knee joints at 60 days $\left(F^{\prime}, G^{\prime}\right)$ and 180 days ( $\left.F^{\prime \prime}-G^{\prime \prime}\right)$ post induction. $\left(\mathbf{H}-\mathrm{H}^{\prime}\right)$ Toluidine blue staining in vehicle control $(\mathrm{H})$ and TAM injected knee joints at 180 days $\left(\mathrm{H}^{\prime}\right)$ post induction. (II') Immunoreactivity for NITEGEin vehicle control (I) and TAM injected knee joints at 180

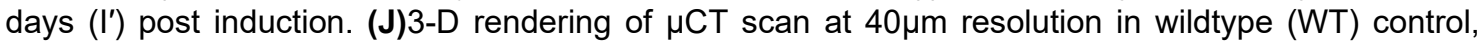
vehicle control and TAM injected knee joint at180 days post induction, black arrows show osteophytes. $\mathrm{n}=4$ per group. Scale bar $=100 \mu \mathrm{m}$.

Fig. 2. BMP signaling induced transient cartilage differentiation is necessary for the pathogenesis of OA.

(A) Schematic representation depicting the generation ofBmp2 $2^{c / c} ; B m p 4^{c / c}$; Col2a1-Cre-ERT2 and the regimen for depletion of BMP signaling by administration of tamoxifen followed by ACLT. (B-H") Longitudinal sections through the knee joints of sham $(B-H)$, "ACLT+vehicle" control $\left(B^{\prime}-H^{\prime}\right)$ and "BMP depletion+ACLT" (B"-H") mice at 56 days post-surgery (PS56). Immunoreactivity for pSMAD1/5/8 (B$\left.B^{\prime \prime}\right)$, Colll (C-C"), ColX (D-D"), MMP-13 (E-E"), NITEGE (G-G") and Coll (H-H") levels. (F-F") Safranin $\mathrm{O}$ staining. (I-I") 3-D rendering of $\mu \mathrm{CT}$ at PS56. Red arrowheads indicate osteophytes, surface roughness, and damage. $n=4$ per time point per group. Scale bar $=100 \mu \mathrm{m}$. (J-K'") Histological sections of knee articular cartilage from OA patients $(n=6)\left(J-J J^{\prime \prime}\right)$, and a patient without known history of knee OA $(n=1)\left(K-K^{\prime \prime \prime}\right)$. $(J, K)$ Safranin O/Fast Green staining of $O A(J)$ and normal $(K)$ cartilage. Immunoreactivity for pSMAD1/5/8 with $\left(\mathrm{J}^{\prime}, \mathrm{K}^{\prime}\right)$ or without phosphatase pre-treatment to verify antibody specificity $\left(\mathrm{J}^{\prime \prime}, \mathrm{J}^{\prime \prime \prime}, \mathrm{K}^{\prime \prime}, \mathrm{K}^{\prime \prime \prime}\right)$, of OA $\left(\mathrm{J}^{\prime}-\mathrm{J}^{\prime \prime \prime}\right)$ and normal $\left(\mathrm{K}^{\prime}-\mathrm{K}^{\prime \prime \prime}\right)$ cartilage. $\left(\mathrm{J}^{\prime \prime}, \mathrm{K}^{\prime \prime \prime}\right)$ Higher magnification view of the marked regions in $\mathrm{J}^{\prime \prime}$ and $\mathrm{K}^{\prime \prime}$. Scale bar $=50 \mu \mathrm{m}$.

The panels where $B m p 2 / 4$ depleted animals were subjected to ACLT are marked as "BMP depletion + ACLT". Vehicle injected animals were used as genotype controls ("ACLT + Vehicle". "Sham" refers toBmp2 ${ }^{c / c} ; B m p 4^{c / c} ;$ Col2a1-Cre-ERT2 animals which underwent sham surgery without ACLT.

Fig. 3. Local pharmacological inhibition of BMP signaling halts OA progression in mice.

(A) Schematic for local inhibition of BMP signaling using LDN-193189 in surgically induced OA in wildtype mice. (B-H") Longitudinal sections through the knee joints of sham (B-H), "ACLT + vehicle" control (B'-H') and "ACLT + LDN-193189" (B"-H") mice at 28 days post-surgery (PS28). Immunoreactivity for pSMAD1/5/8 (B-B"), Colll (C-C"), ColX (D-D"), MMP-13 (E-E"), NITEGE (F-F") and Coll (H-H") levels. (G-G") Safranin O staining. (I-I") 3-D rendering of $\mu C T$ scan at resolution of $5.86 \mu \mathrm{m}$ per pixel in sham (I), "ACLT + vehicle" control (I') and "ACLT + LDN-193189" injected (I") knee joint atPS28. Red arrows show osteophytes. (J) OARSI severity score for "ACLT + vehicle" vs "ACLT + LDN-193189" and "ACLT + vehicle" vs Sham. Scale bar $=100 \mu \mathrm{m}, \mathrm{n}=4$ per group. ${ }^{* * * *} \mathrm{p}<$ 0.0001 .

Fig. 4. Inhibition of BMP signaling post onset of surgically induced $O A$ attenuates the severity of OA associated changes.

(A) Schematic for local inhibition of BMP signaling, using of LDN-193189, in the knee joint of wildtype mouse post-surgical onset of OA. (B-F") Longitudinal sections through the knee joints of sham (B-F), "ACLT + vehicle" control ( $\left.B^{\prime}-F^{\prime}\right)$ and "ACLT + LDN-193189" (B"-F") mice at 56 days post-surgery (PS56). Immunoreactivity for ColX (B-B"), MMP-13 (C-C"), NITEGE (D-D") and Coll (F-F") levels. White dashed lines mark the bone ends of femur and tibia. (E-E") Toluidine blue staining. (G-G") 3-D rendering of $\mu C T$ scan at resolution of $5.86 \mu \mathrm{m}$ per pixel in sham, "ACLT + vehicle" control and "ACLT 
+ LDN-193189" injected knee joint atPS56. Red arrows mark osteophytes. (H) OARSI severity score for "ACLT + vehicle" vs "ACLT + LDN-193189", " $p=0.0135$, "ACLT + vehicle" vs Sham, ${ }^{* * *} p=$ 0.0007 . Scale bar $=100 \mu \mathrm{m}, \mathrm{n}=3$ per group,

Fig. 5. Local inhibition of BMP signaling post-onset of surgically induced OA attenuates the severity of $O A$ associated changes for longer duration.

Schematic for local inhibition of BMP signaling, using of LDN-193189, in the knee joint of wildtype mouse post-surgical onset of OA. (A). Longitudinal sections through the knee joints of ACLT induced OA mice (B-D"'). "ACLT + vehicle" control (B, C and D), "ACLT + LDN-193189 one dose" (B', C' and $\left.D^{\prime}\right)$, "ACLT + LDN-193189 two doses" (B", C" and D"), "ACLT + LDN-193189 three doses" (B"', C"' and $\left.\mathrm{D}^{\prime \prime \prime}\right)$ mice at 84 days post ACLT. Immunoreactivity for Colll (B- B'"'), ColX (C- C'"') and Safranin O staining (D- $\left.D^{\prime \prime \prime}\right)$. Immunoreactivity for SMURF1 in control (E), post ACLT 30 days ( $\left.E^{\prime}\right)$ and 60 days $\left(E^{\prime \prime}\right)$. Scale bar $=100 \mu \mathrm{m}, \mathrm{n}=3$ per group.

Fig. 6. Effect of local inhibition of BMP signaling on inflammatory responses in a surgically induced osteoarthritic mouse model.

(A-D") Longitudinal sections through the knee joints of sham (A-D), "ACLT + vehicle" control (A'-D') and "ACLT + LDN-193189" (A"-D") mice at 56 days post-surgery (PS56). Immunoreactivity for IL-1 $\beta$ $\left(A-A^{\prime \prime}\right)$, iNOS (B-B"), NF-kB (C-C") and TNF- $\alpha$ (D-D") levels. White arrows indicate TNF- $\alpha$ positive cells. $n=4$ per group. Scale bar $=100 \mu \mathrm{m}$.

Fig. S1. Molecular characterization of mouse knee joint post ACLT

(A-D'")Longitudinal sections through the knee joints of sham (A, C), 7 days (B, D), 14 days (B', $\left.D^{\prime}\right), 21$ days (B", D"), 28 days (B"', D"') post ACLT. (A-B"') Immunoreactivity for pSMAD1/5/8. (C-D"')BrdU uptake. Insets display the higher magnification view of the marked regions.

Fig. S2. BMP signaling induced transient cartilage differentiation is necessary for the pathogenesis of OA.

(A) Schematic representation depicting the generation ofBmp2/c; Bmp4/c; Col2a1-Cre-ERT2 and the regimen for depletion of BMP signaling by administration of tamoxifen followed by ACLT. (B-G") Longitudinal sections through the knee joints of sham (B-G), "ACLT + vehicle control" $\left(B^{\prime}-G^{\prime}\right)$ and "BMP depletion + ACLT" (B"-G") mice at 28 days post-surgery (PS28). Immunoreactivity for pSMAD1/5/8 (B-B"), Colll (C-C"), ColX (D-D"), MMP-13 (E-E"), NITEGE (G-G"). (F-F") Safranin O staining. $\mathrm{n}=4$ per group. Scale bar $=100 \mu \mathrm{m}$.

The panels where Bmp2/4 depleted animals were subjected to ACLT are marked as "BMP depletion + ACLT". Vehicle injected animals were used as genotype controls ("ACLT + Vehicle".

Fig. S3.Determination of optimum LDN-193189 concentration to inhibit BMP signaling using BRITER (BMP Responsive Immortalized Reporter) cell line.

Normalized relative luciferase activity after $3 \mathrm{~h}$ of LDN-193189 treatment with different concentration (100nM, 200nM, 400nM) to inhibit BMP signaling in BRITER cell line (Fig.S3). 
1. Ray A, Singh PN, Sohaskey ML, Harland RM, \& Bandyopadhyay A (2015) Precise spatial restriction of BMP signaling is essential for articular cartilage differentiation. Development 142(6):1169-1179.

2. Mankin HJ (1963) Localization of Tritiated Thymidine in Articular Cartilage of Rabbits: III. MATURE ARTICULAR CARTILAGE. JBJS 45(3):529-540.

3. Roelofs AJ \& De Bari C (2019) Immunostaining of Skeletal Tissues. Methods Mol Biol 1914:437-450.

4. Vos T, et al. (2012) Years lived with disability (YLDs) for 1160 sequelae of 289 diseases and injuries 1990-2010: a systematic analysis for the Global Burden of Disease Study 2010. Lancet 380(9859):2163-2196.

5. Cross M, et al. (2014) The global burden of hip and knee osteoarthritis: estimates from the global burden of disease 2010 study. Annals of the rheumatic diseases 73(7):1323-1330.

6. Palazzo C, Nguyen C, Lefevre-Colau MM, Rannou F, \& Poiraudeau S (2016) Risk factors and burden of osteoarthritis. Annals of physical and rehabilitation medicine 59(3):134-138.

7. Chen D, et al. (2017) Osteoarthritis: toward a comprehensive understanding of pathological mechanism. Bone research 5:16044.

8. Kozhemyakina E, Lassar AB, \& Zelzer E (2015) A pathway to bone: signaling molecules and transcription factors involved in chondrocyte development and maturation. Development 142(5):817-831.

9. Ortega N, Behonick DJ, \& Werb Z (2004) Matrix remodeling during endochondral ossification. Trends in cell biology 14(2):86-93.

10. Long F \& Ornitz DM (2013) Development of the endochondral skeleton. Cold Spring Harbor perspectives in biology 5(1):a008334.

11. Dahlberg L, et al. (2000) Selective enhancement of collagenase-mediated cleavage of resident type II collagen in cultured osteoarthritic cartilage and arrest with a synthetic inhibitor that spares collagenase 1 (matrix metalloproteinase 1). Arthritis and rheumatism 43(3):673-682.

12. Neuhold LA, et al. (2001) Postnatal expression in hyaline cartilage of constitutively active human collagenase-3 (MMP-13) induces osteoarthritis in mice. The Journal of clinical investigation 107(1):35-44.

13. Enomoto $\mathrm{H}$, et al. (2003) Vascular endothelial growth factor isoforms and their receptors are expressed in human osteoarthritic cartilage. The American journal of pathology 162(1):171181.

14. Ko JY, et al. (2017) MicroRNA-29a Counteracts Synovitis in Knee Osteoarthritis Pathogenesis by Targeting VEGF. Scientific reports 7(1):3584.

15. Lark MW, et al. (1997) Aggrecan degradation in human cartilage. Evidence for both matrix metalloproteinase and aggrecanase activity in normal, osteoarthritic, and rheumatoid joints. The Journal of clinical investigation 100(1):93-106.

16. Glasson SS, et al. (2005) Deletion of active ADAMTS5 prevents cartilage degradation in a murine model of osteoarthritis. Nature 434(7033):644-648.

17. Song $\mathrm{RH}$, et al. (2007) Aggrecan degradation in human articular cartilage explants is mediated by both ADAMTS-4 and ADAMTS-5. Arthritis and rheumatism 56(2):575-585.

18. Barranco C (2014) Osteoarthritis: Animal data show VEGF blocker inhibits post-traumatic OA. Nature reviews. Rheumatology 10(11):638.

19. He $Y$, et al. (2014) Type $X$ collagen levels are elevated in serum from human osteoarthritis patients and associated with biomarkers of cartilage degradation and inflammation. BMC musculoskeletal disorders 15:309.

20. Duprez D, et al. (1996) Overexpression of BMP-2 and BMP-4 alters the size and shape of developing skeletal elements in the chick limb. Mechanisms of development 57(2):145-157. 
21. Zou H, Wieser R, Massague J, \& Niswander L (1997) Distinct roles of type I bone morphogenetic protein receptors in the formation and differentiation of cartilage. Genes \& development 11(17):2191-2203.

22. Singh PNP, et al. (2018) Precise spatial restriction of BMP signaling in developing joints is perturbed upon loss of embryo movement. Development 145(5).

23. Kahn J, et al. (2009) Muscle contraction is necessary to maintain joint progenitor cell fate. Developmental cell 16(5):734-743.

24. Rolfe RA, et al. (2014) Identification of mechanosensitive genes during skeletal development: alteration of genes associated with cytoskeletal rearrangement and cell signalling pathways. BMC genomics 15:48.

25. Mankin HJ (1962) Localization of Tritiated Thymidine in Articular Cartilage of Rabbits: I. Growth In Immature Cartilage. JBJS 44(4):682-688.

26. Schmal H, et al. (2012) Expression of BMP-receptor type 1A correlates with progress of osteoarthritis in human knee joints with focal cartilage lesions. Cytotherapy 14(7):868-876.

27. Nakase T, et al. (2003) Localization of bone morphogenetic protein-2 in human osteoarthritic cartilage and osteophyte. Osteoarthritis Cartilage 11(4):278-284.

28. Hayami T, et al. (2006) Characterization of articular cartilage and subchondral bone changes in the rat anterior cruciate ligament transection and meniscectomized models of osteoarthritis. Bone 38(2):234-243.

29. Glasson SS, Blanchet TJ, \& Morris EA (2007) The surgical destabilization of the medial meniscus (DMM) model of osteoarthritis in the 129/SvEv mouse. Osteoarthritis and cartilage /OARS, Osteoarthritis Research Society 15(9):1061-1069.

30. Bandyopadhyay A, et al. (2006) Genetic analysis of the roles of BMP2, BMP4, and BMP7 in limb patterning and skeletogenesis. PLoS genetics 2(12):e216.

31. Yu PB, et al. (2008) BMP type I receptor inhibition reduces heterotopic [corrected] ossification. Nature medicine 14(12):1363-1369.

32. Hao J, et al. (2010) In vivo structure-activity relationship study of dorsomorphin analogues identifies selective VEGF and BMP inhibitors. ACS chemical biology 5(2):245-253.

33. Sanvitale CE, et al. (2013) A new class of small molecule inhibitor of BMP signaling. PloS one 8(4):e62721.

34. Mankin HJ (1962) Localization of Tritiated Thymidine in Articular Cartilage of Rabbits: II. Repair in Immature Cartilage. JBJS 44(4):688-698.

35. Kamekura S, et al. (2005) Osteoarthritis development in novel experimental mouse models induced by knee joint instability. Osteoarthritis and cartilage / OARS, Osteoarthritis Research Society 13(7):632-641.

36. Kobayashi M, et al. (2005) Role of interleukin-1 and tumor necrosis factor alpha in matrix degradation of human osteoarthritic cartilage. Arthritis and rheumatism 52(1):128-135.

37. Tetlow LC, Adlam DJ, \& Woolley DE (2001) Matrix metalloproteinase and proinflammatory cytokine production by chondrocytes of human osteoarthritic cartilage: associations with degenerative changes. Arthritis and rheumatism 44(3):585-594.

38. Lin AC, et al. (2009) Modulating hedgehog signaling can attenuate the severity of osteoarthritis. Nature medicine 15(12):1421-1425.

39. Zhou J, et al. (2014) Disrupting the Indian hedgehog signaling pathway in vivo attenuates surgically induced osteoarthritis progression in Col2a1-CreERT2; Ihhfl/fl mice. Arthritis research \& therapy 16(1):R11.

40. Zhou J, Wei X, \& Wei L (2014) Indian Hedgehog, a critical modulator in osteoarthritis, could be a potential therapeutic target for attenuating cartilage degeneration disease. Connective tissue research 55(4):257-261.

41. Vortkamp A, et al. (1996) Regulation of rate of cartilage differentiation by Indian hedgehog and PTH-related protein. Science 273(5275):613-622. 
42. Mak KK, Kronenberg HM, Chuang PT, Mackem S, \& Yang Y (2008) Indian hedgehog signals independently of PTHrP to promote chondrocyte hypertrophy. Development 135(11):19471956.

43. Gelse K, et al. (2012) Molecular differentiation between osteophytic and articular cartilage-clues for a transient and permanent chondrocyte phenotype. Osteoarthritis and cartilage / OARS, Osteoarthritis Research Society 20(2):162-171.

44. Pitsillides AA \& Beier F (2011) Cartilage biology in osteoarthritis--lessons from developmental biology. Nature reviews. Rheumatology 7(11):654-663.

45. Sandell LJ \& Aigner T (2001) Articular cartilage and changes in arthritis. An introduction: cell biology of osteoarthritis. Arthritis research 3(2):107-113.

46. Sokolove J \& Lepus CM (2013) Role of inflammation in the pathogenesis of osteoarthritis: latest findings and interpretations. Therapeutic advances in musculoskeletal disease 5(2):7794.

47. Goldring MB \& Otero M (2011) Inflammation in osteoarthritis. Current opinion in rheumatology 23(5):471-478.

48. Ye T \& Yi Y (2017) Sample size calculations in clinical research, by Shein-Chung Chow, Jun Shao, Hansheng Wang, and Yuliya Lokhnygina: Chapman \& Hall/CRC Biostatistics Series, New York, Taylor \& Francis, 2017, 510 pp., \$99.95 (hardback), ISBN: 978-1-138-74098-3. (Taylor \& Francis).

49. Muzumdar MD, Tasic B, Miyamichi K, Li L, \& Luo L (2007) A global double-fluorescent Cre reporter mouse. Genesis 45(9):593-605. 

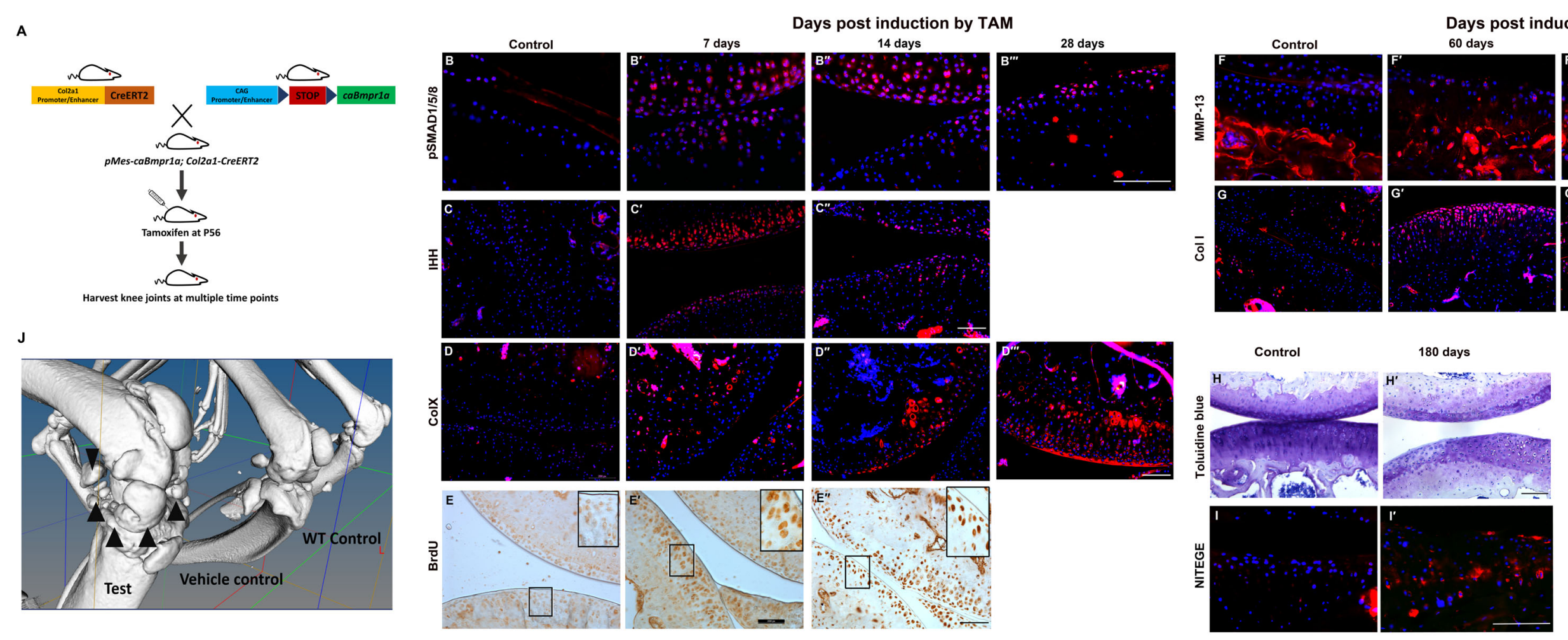

Fig. 1

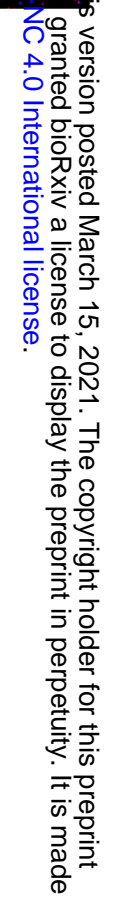


bioRxiv preprint doi: https://doi.org/10.1101/2021.03.01.433366; this version posted March 15, 2021. The copyright holder for this preprint (which was not certified by peer review) is the author/funder, who has granted bioRxiv a license to display the preprint in perpetuity. It is made available under aCC-BY-NC 4.0 International license.

A

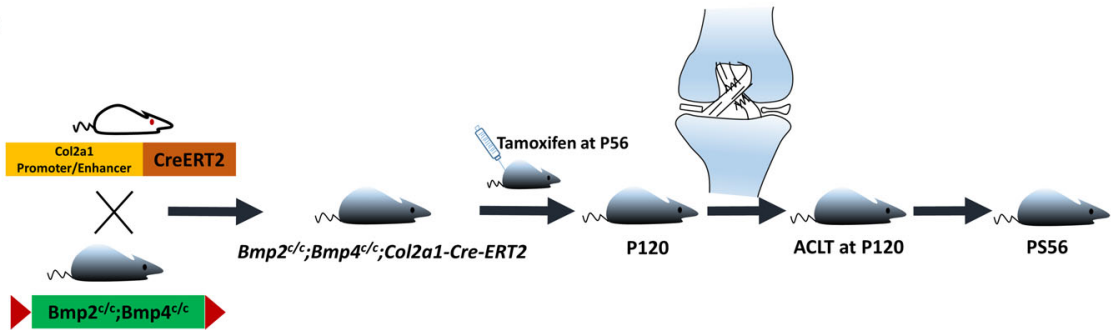

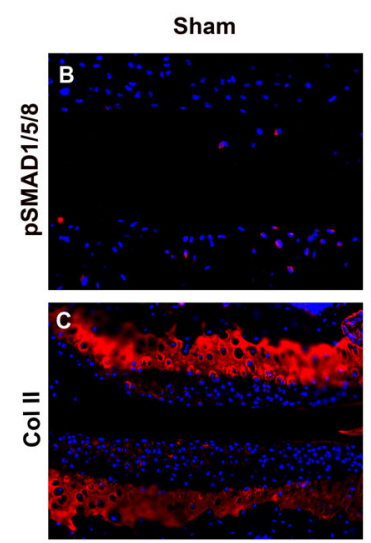

ACLT + VEHICLE

BMP DEPLETION + ACLT
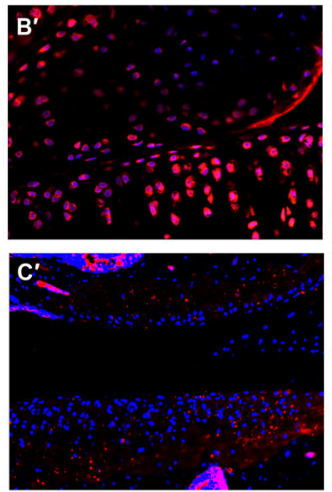

응

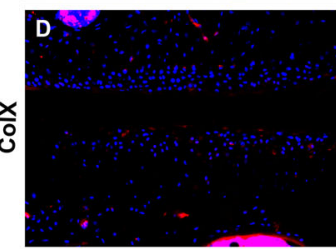

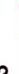
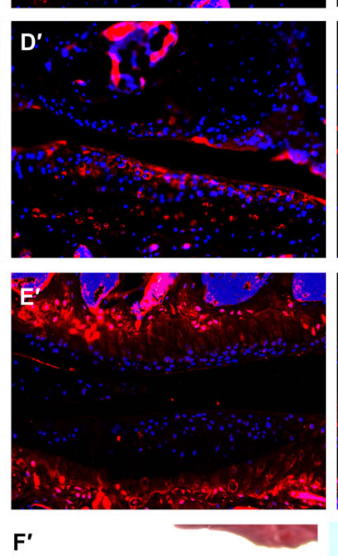
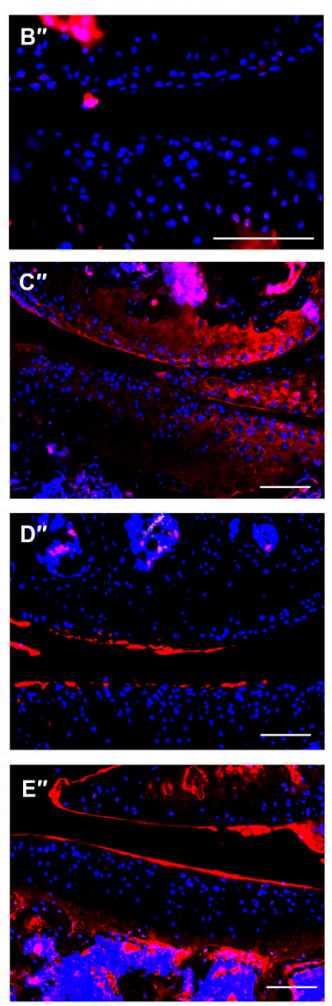

F"

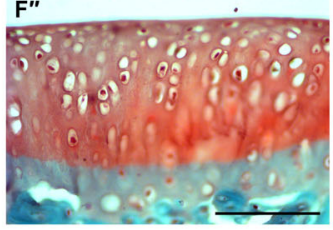

Sham

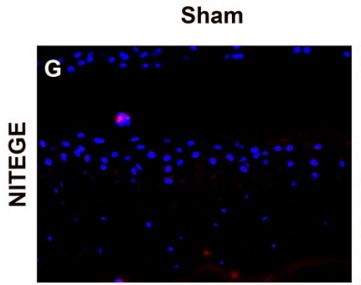

ACLT + VEHICLE
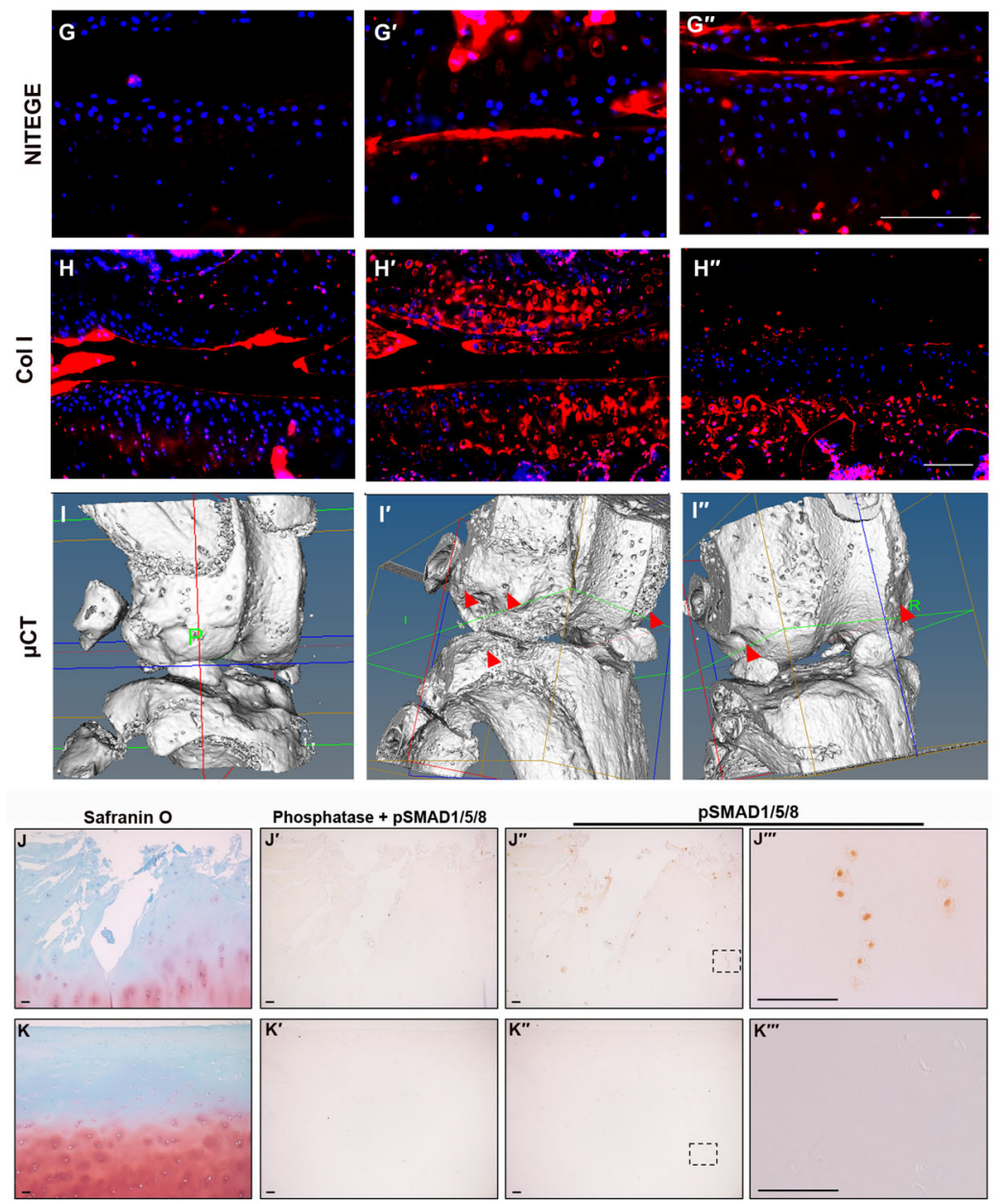

Fig. 2 
bioRxiv preprint doi: https://doi.org/10.1101/2021.03.01.433366; this version posted March 15, 2021. The copyright holder for this preprint (which was not certified by peer review) is the author/funder, who has granted bioRxiv a license to display the preprint in perpetuity. It is made available under aCC-BY-NC 4.0 International license.

A

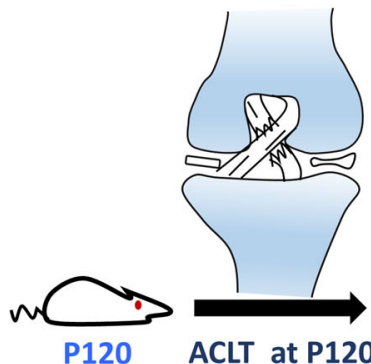

Sham

选
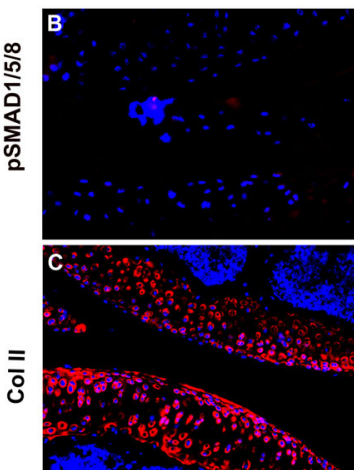

음

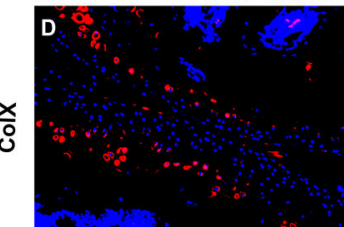



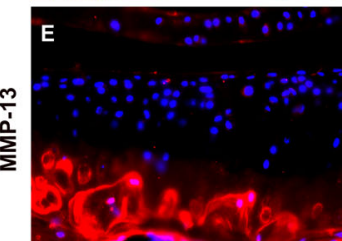

岂

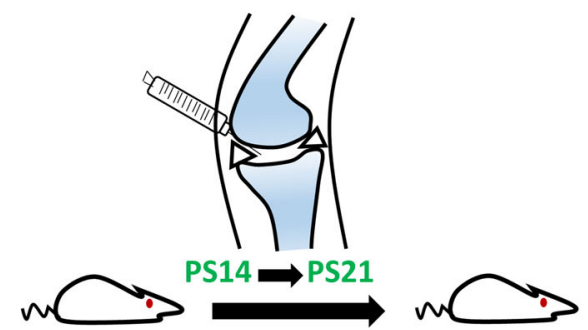

LDN-193189

PS28

(intra-articular)
ACLT + LDN -193189
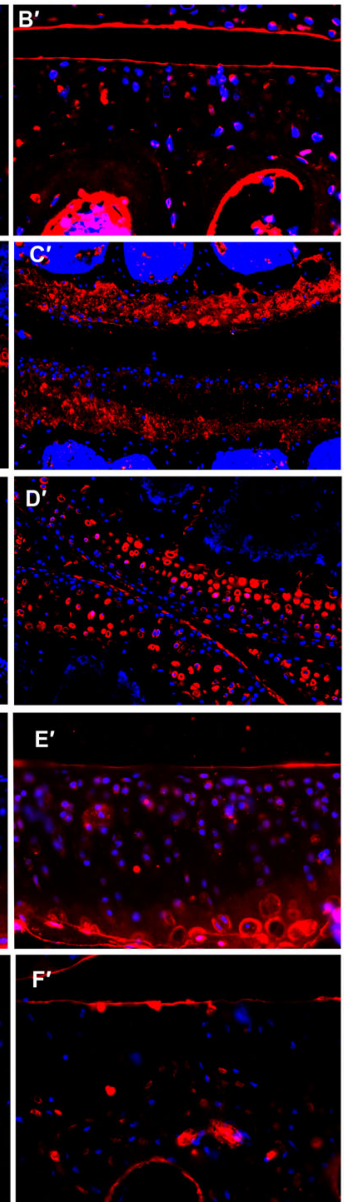
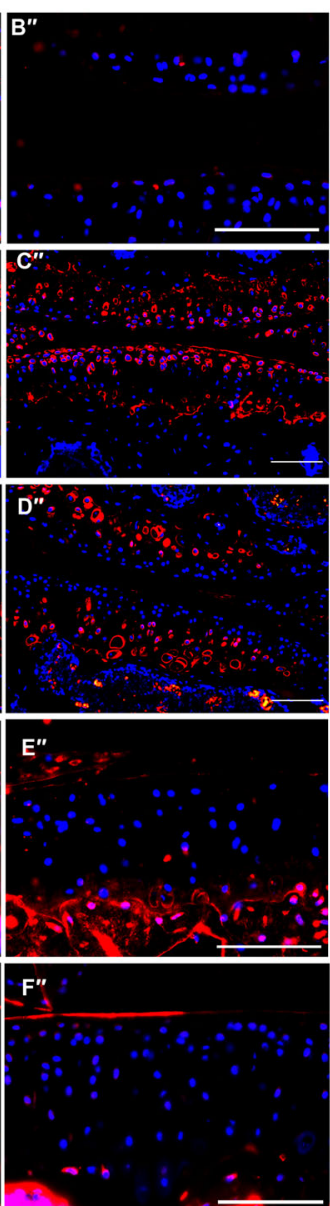

Sham
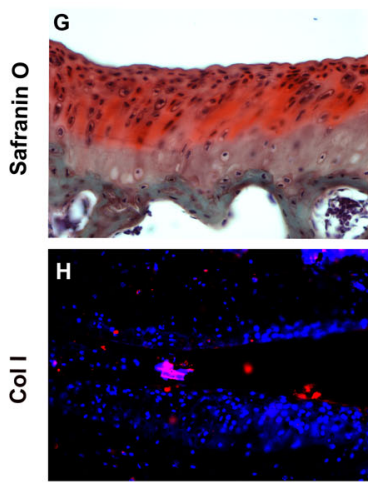

ACLT + Vehicle

G'
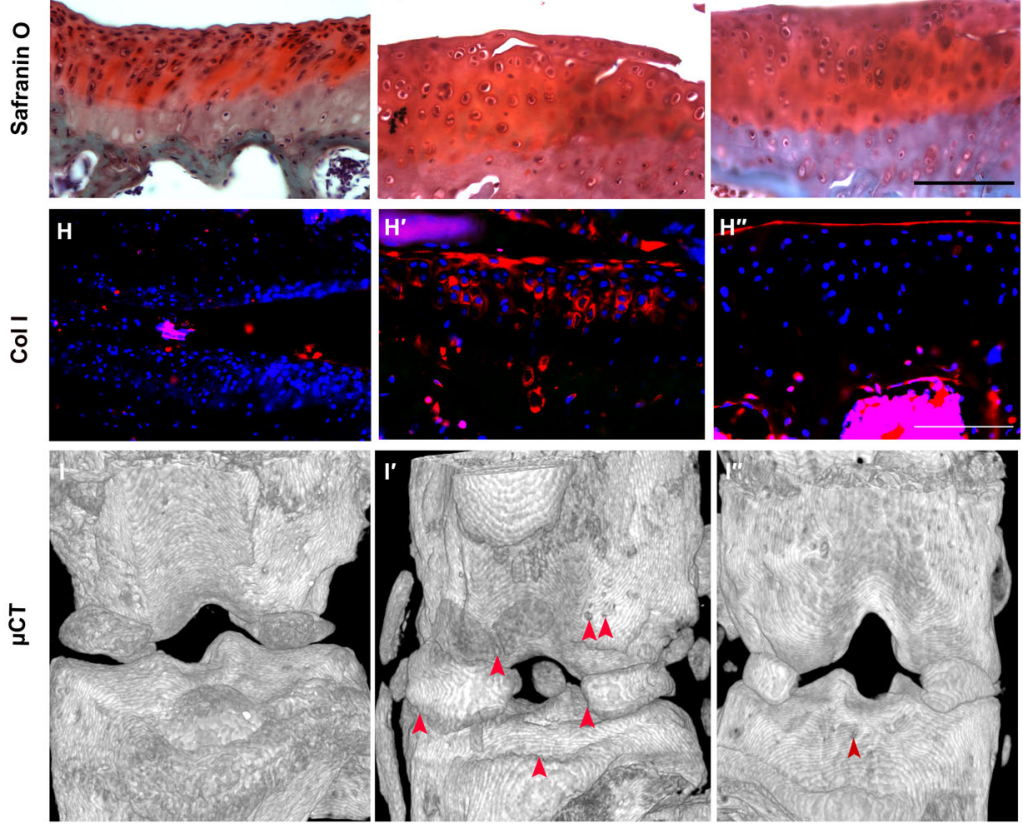

J

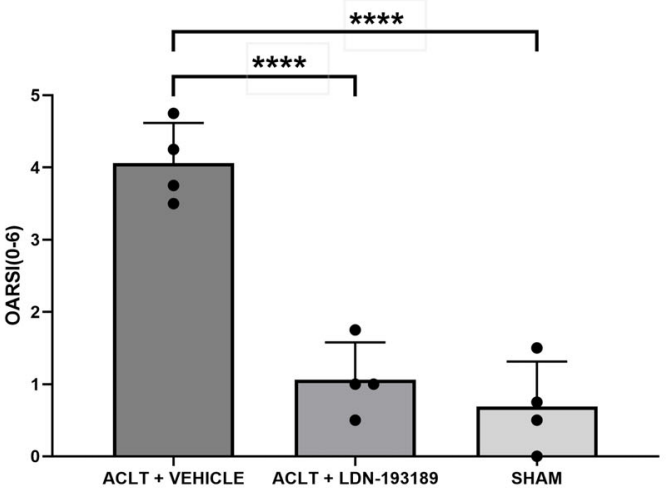

ב $\mathrm{ACLT}+\mathrm{VEHICLE}$

ㅁ $\mathrm{ACLT}+\mathrm{LDN}-193189$

口 SHAM

Fig. 3 
bioRxiv preprint doi: https://doi.org/10.1101/2021.03.01.433366; this version posted March 15, 2021. The copyright holder for this preprint (which was not certified by peer review) is the author/funder, who has granted bioRxiv a license to display the preprint in perpetuity. It is made available under aCC-BY-NC 4.0 International license.

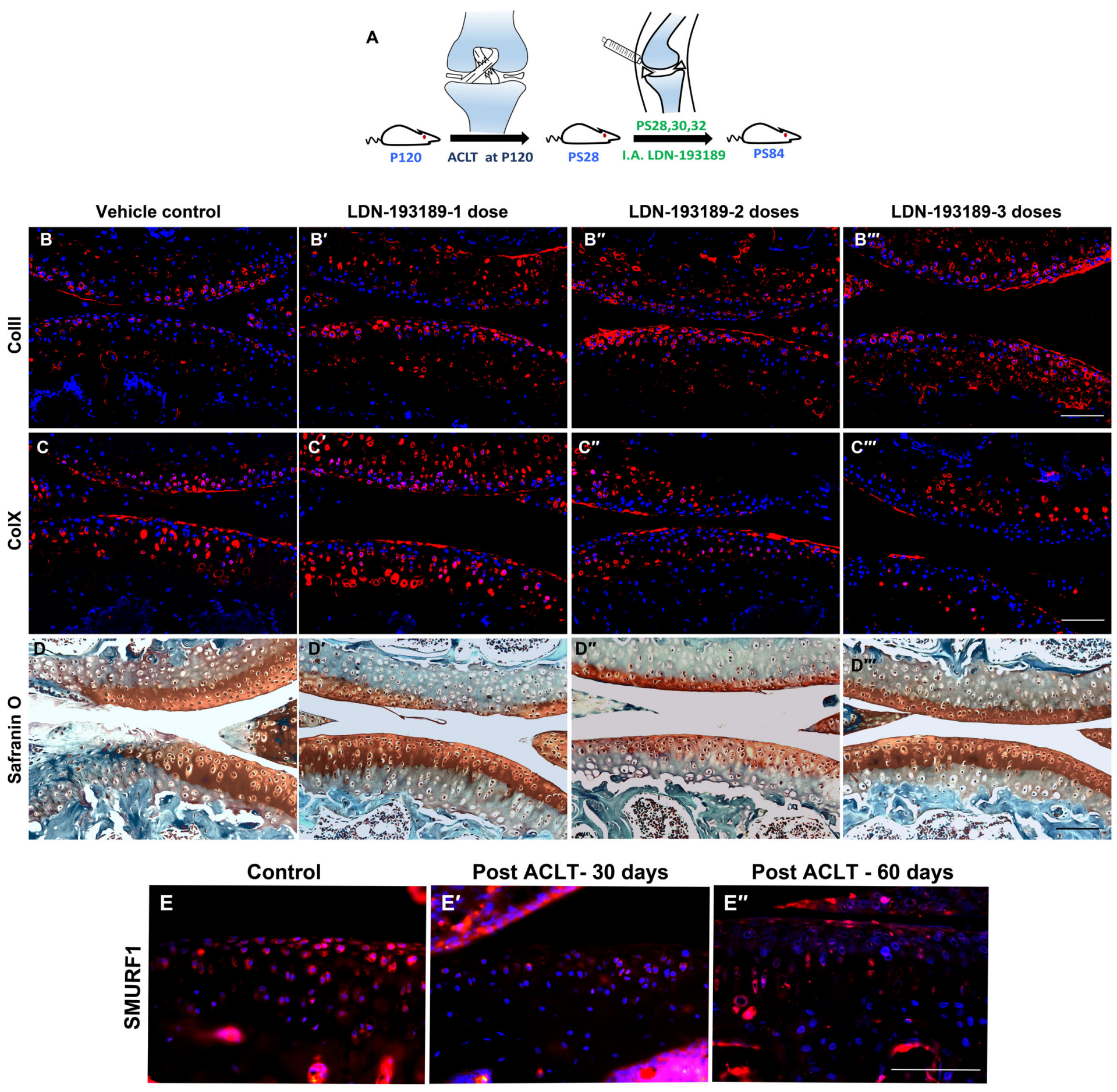

Fig. 5 
Sham
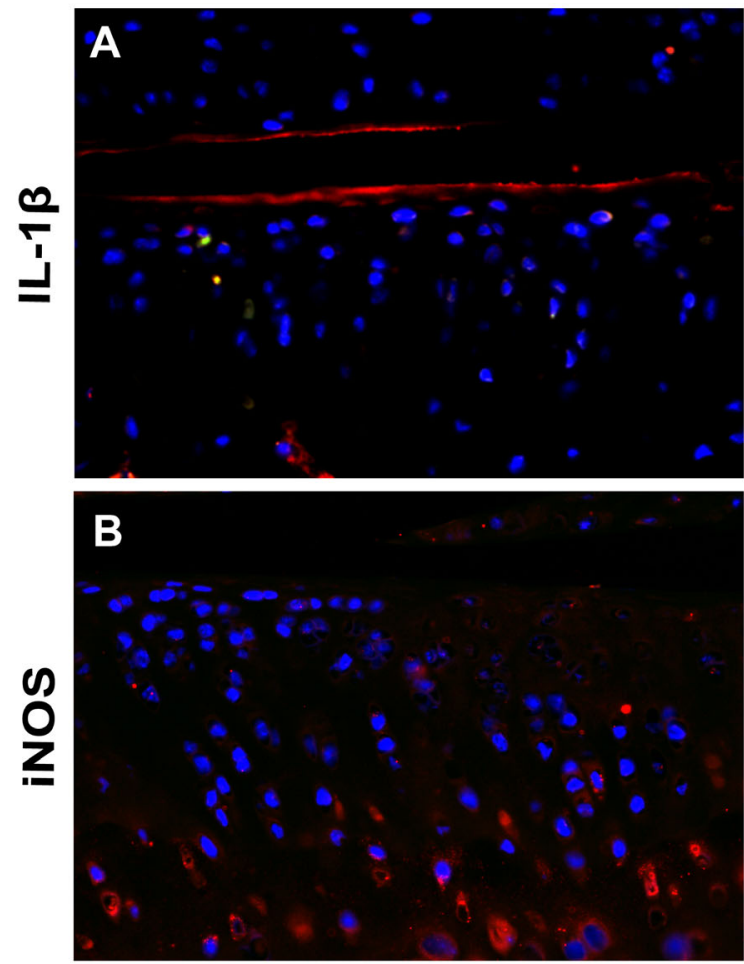

$\frac{m}{y}$

C

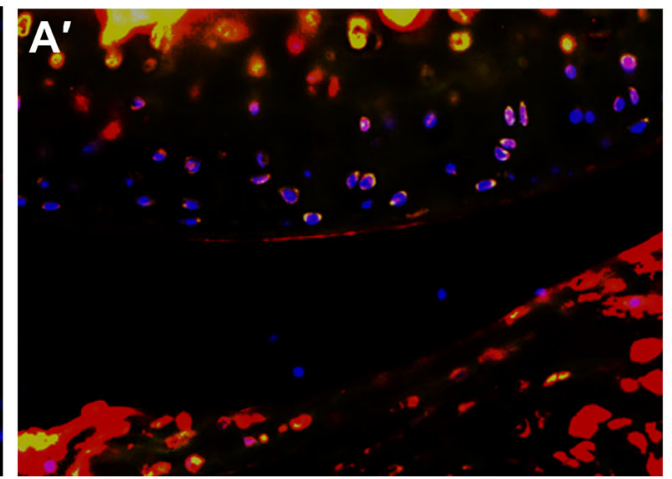

18
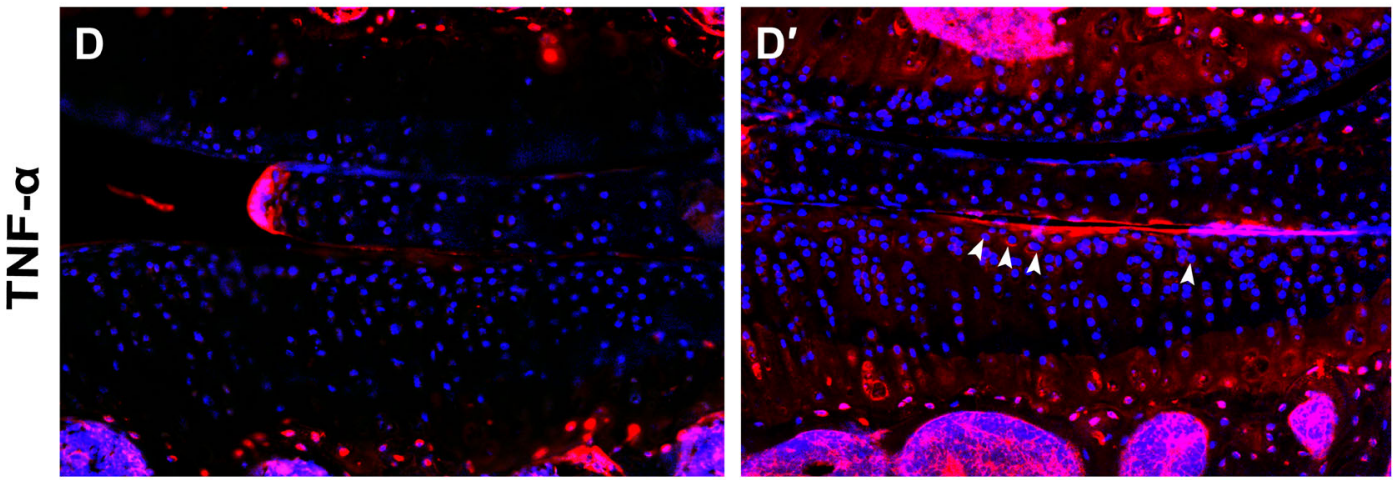

ACLT + Vehicle

B'

4.8?

Nos 4 -

$\mathbf{C}^{\prime}$

6

ACLT + LDN-193189

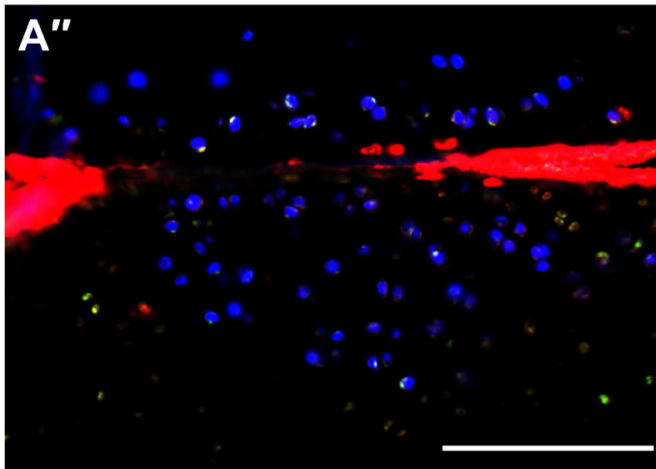

\section{B"}
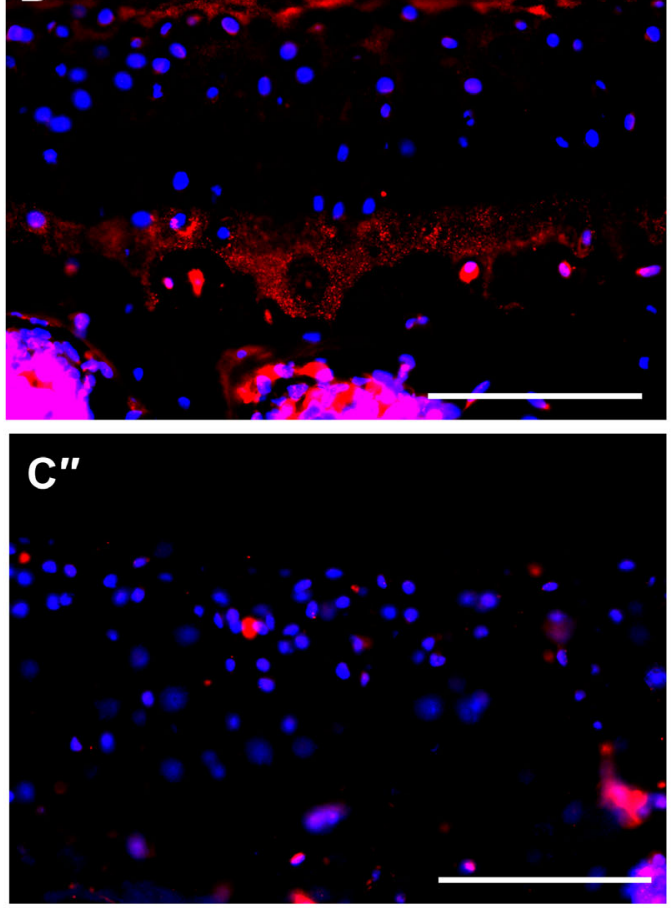

D'

44

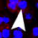

$D^{\prime \prime}$

(8)

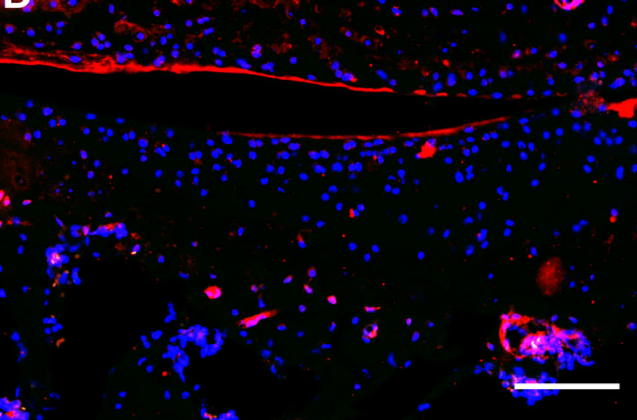

Fig. 6 
Days post ACLT
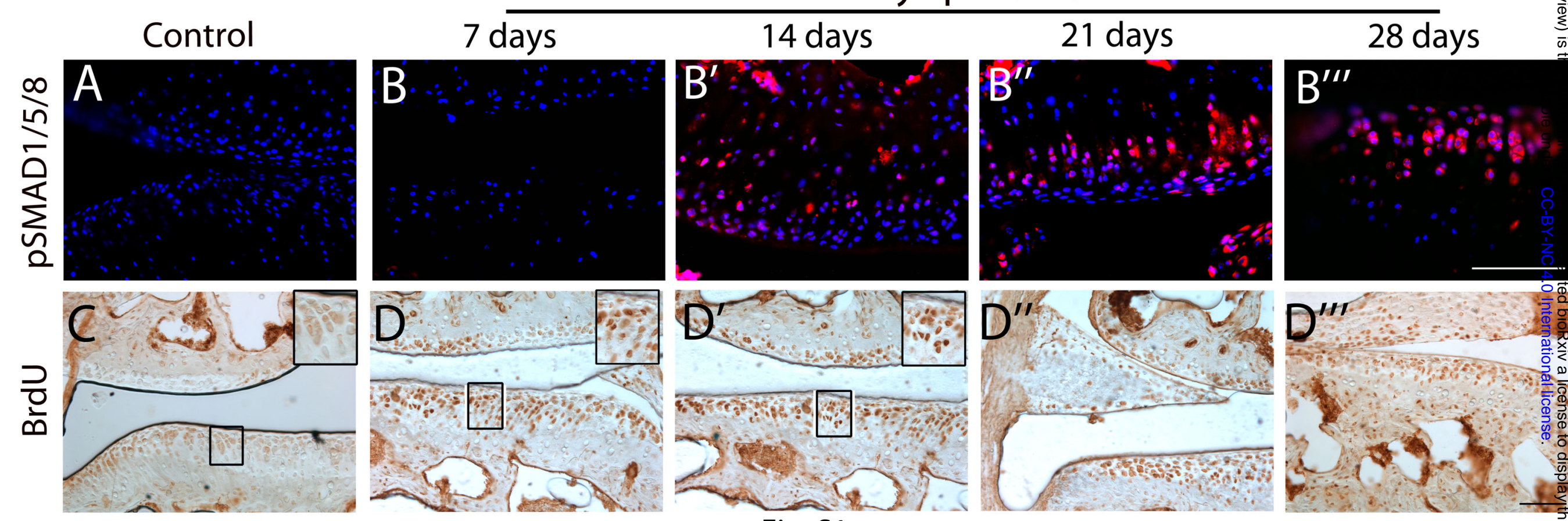

Fig. S1
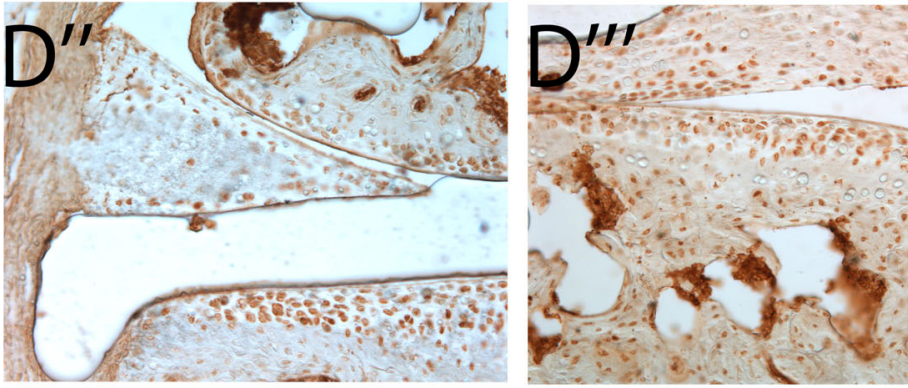
bioRxiv preprint doi: https://doi.org/10.1101/2021.03.01.433366; this version posted March 15, 2021. The copyright holder for this preprint (which was At certified by peer review) is the author/funder, who has granted bioRxiv a license to display the preprint in perpetuity. It is made available under aCC-BY-NC 4.0 International license.
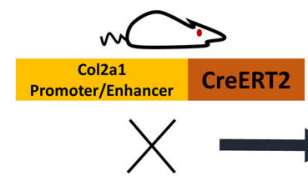

$B m p 2^{c / c} ; B m p 4^{c / c}$
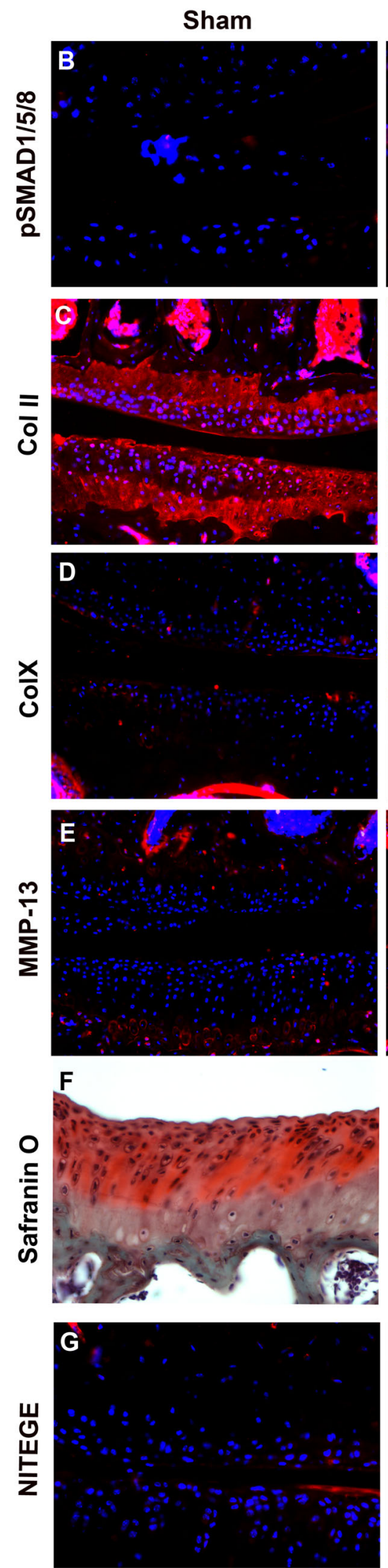

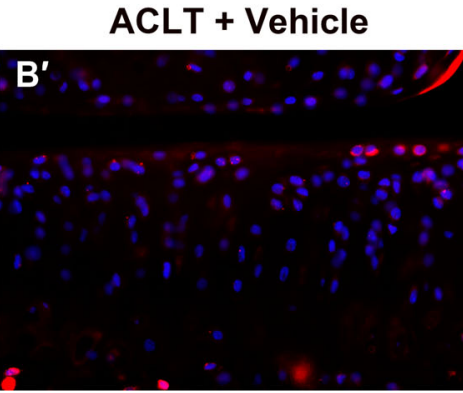

\section{BMP depletion + ACLT}
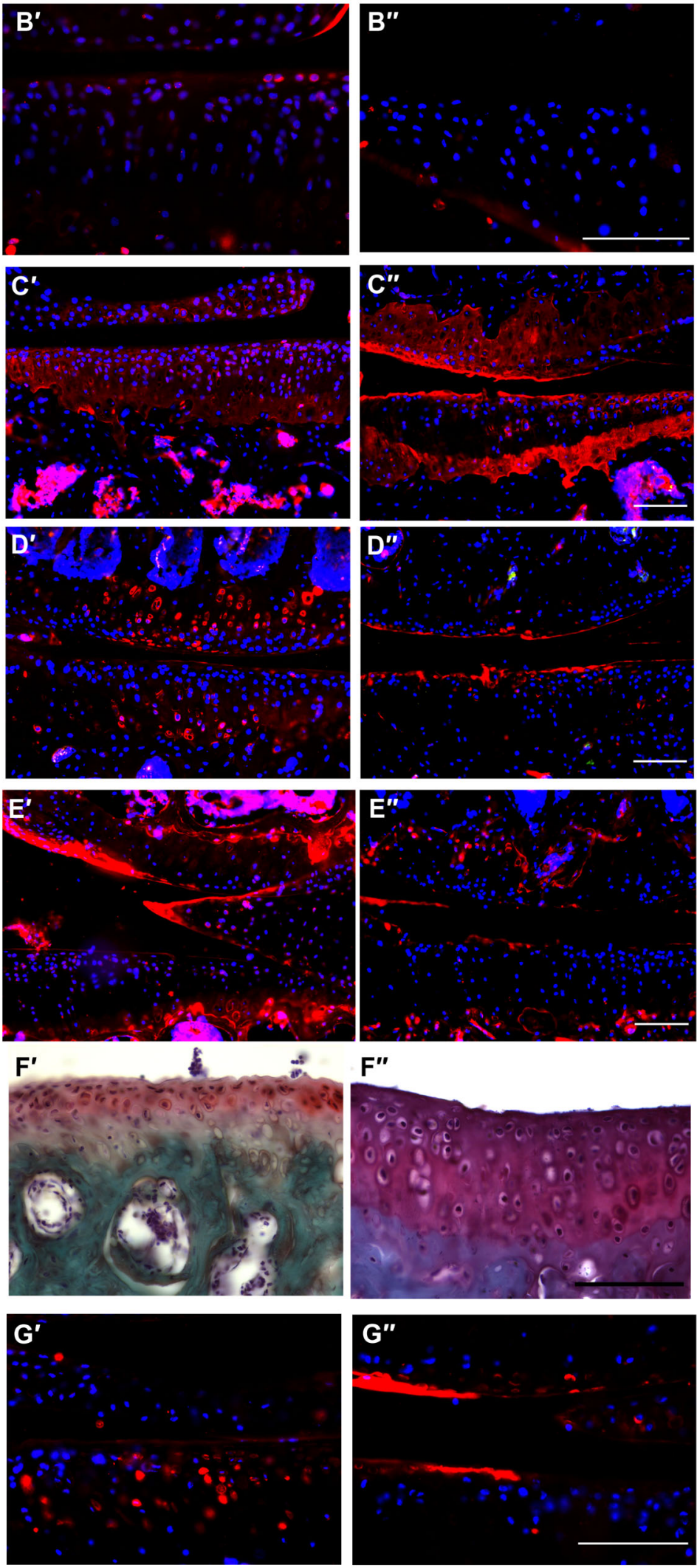

Fig. S2 


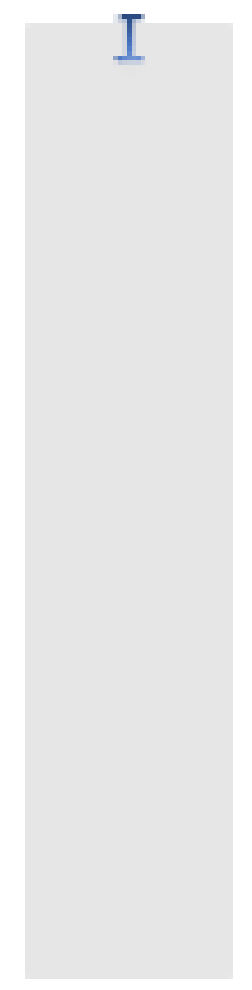

Untreated

rhBMP2

rhBMP2+

LDN193189

rhBMP2+

rhBMP2+

(100nM)

LDN193189

LDN193189 (200nM)

(400nM)

Fig. S3 